\title{
Supplementary Materials: Bayesian Sparse Multivariate Regression with Asymmetric Nonlocal Priors for Microbiome Data Analysis
}

Kurtis Shuler*, Marilou Sison-Mangus ${ }^{\dagger}$ and Juhee Lee*

\section{MCMC Algorithm}

We obtain a sample from the posterior distribution using an MCMC comprised of a combination of Gibbs and Metropolis-within-Gibbs steps. Recall that we have mixtureof-mixtures distributions for the priors of $r_{t k}$ and $\alpha_{0 j}$ in (5) and (6) of the main text, respectively. For easy posterior simulation, we introduce latent variables that indicate which mixture component $r_{t k}$ and $\alpha_{0 j}$ are from, and do categorical/Bernoulli draws to update these latent variables. Specifically, for $r_{t k}$, we let $c_{t k}^{r}=\ell, \ell=1, \ldots, L^{r}$ if and only if $r_{t k}$ came from the $\ell^{t h}$ mixture component. Conditional on $c_{t k}^{r}$, we introduce another indicator variable $\lambda_{t k}^{r} \in\{0,1\}$ to indicate the Gaussian component from which $r_{t k}$ came, $\mathrm{N}\left(\eta_{\ell}^{r}, u_{r}^{2}\right)$ or $\mathrm{N}\left(\frac{v_{r}-w_{\ell}^{r} \eta_{\ell}^{r}}{1-w_{\ell}^{r}}, u_{r}^{2}\right)$. Let $\mathrm{P}\left(c_{t k}^{r}=\ell \mid \boldsymbol{\psi}^{r}\right)=\psi_{\ell}^{r}$ and $\mathrm{P}\left(\lambda_{t k}^{r}=1 \mid c_{t k}^{r}=\right.$ $\left.\ell, w_{\ell}^{r}\right)=w_{\ell}^{r}$. The joint prior distribution of $\boldsymbol{r}, \boldsymbol{c}^{r}$ and $\boldsymbol{\lambda}^{r}$ can be written as

$$
\begin{aligned}
\mathrm{P}\left(\boldsymbol{c}^{r}, \boldsymbol{\lambda}^{r}, \boldsymbol{r} \mid \boldsymbol{\psi}^{r}, \boldsymbol{w}^{r}, \boldsymbol{\eta}^{r}\right)= & \prod_{t, k} \mathrm{P}\left(c_{t k}^{r} \mid \boldsymbol{\psi}^{r}\right) \mathrm{P}\left(\lambda_{t k}^{r} \mid c_{t k}^{r}, \boldsymbol{w}^{r}\right) \mathrm{P}\left(r_{t k} \mid c_{t k}^{r}, \lambda_{t k}^{r}, \boldsymbol{\eta}^{r}\right) \\
= & \prod_{\ell=1}^{L^{r}}\left(\psi_{\ell}^{r}\right)^{d_{\ell}^{r}} \prod_{t, k}\left(w_{c_{t k}}^{r}\right)^{\lambda_{t k}^{r}}\left(1-w_{c_{t k}}^{r}\right)^{1-\lambda_{t k}^{r}} \\
& \times \prod_{t, k} \mathrm{~N}\left(r_{t k} \mid \eta_{c_{t k}}^{r}, u_{r}^{2}\right)^{\lambda_{t k}^{r}} \mathrm{~N}\left(r_{t k} \mid \frac{v_{r}-w_{c_{t k}}^{r} \eta_{c_{t k}}^{r}}{1-w_{c_{t k}}^{r}}, u_{r}^{2}\right)^{1-\lambda_{t k}^{r}}
\end{aligned}
$$

where $d_{\ell}^{r}=\sum_{t} \sum_{k=1}^{K_{i}} \mathbb{I}\left(c_{t k}=\ell\right)$ denotes the number of elements in the $\ell^{t h}$ mixture component. We update mixture locations $\eta_{\ell}^{r}$ and mixture weights $\boldsymbol{\psi}^{r}$ and $w_{\ell}^{r}$ conditional on $\lambda_{t k}^{r}$ and $c_{t k}^{r}$ using Gibbs steps. Similar to the method used to sample $r_{t k}$, we also introduce auxiliary variables $c_{j}^{\alpha} \in\left\{1, \ldots, L^{\alpha}\right\}$ and $\lambda_{j}^{\alpha} \in\{0,1\}$ to specify the mixture components from which $\alpha_{0 j}$ came, and we let $d_{\ell}^{\alpha}=\sum_{j=1}^{J} \mathbb{I}\left(c_{j}^{\alpha}=\ell\right)$ denote the number of elements assigned to the $\ell^{\text {th }}$ mixture component. Again, we do categorical/Bernoulli draws for $c_{j}^{\alpha}$ and $\lambda_{j}^{\alpha}$ and then update $\alpha_{0 j}$ conditional on these assignments, and update mixture locations $\eta_{\ell}^{\alpha}$ and mixture weights $\psi^{\alpha}$ and $w_{\ell}^{\alpha}$ conditional on mixture labels $\lambda_{j}^{\alpha}$ and $c_{j}^{\alpha}$ using Gibbs steps. Letting $\underline{\boldsymbol{\theta}}$ denote the vector of all unknown parameters

\footnotetext{
*Department of Statistics, University of California Santa Cruz, Santa Cruz, CA kshuler@ucsc.edu juheelee@soe.ucsc.edu

${ }^{\dagger}$ Department of Ocean Sciences, University of California Santa Cruz, Santa Cruz, CA msisonma@ucsc.edu
} 
and $\boldsymbol{\Omega}$ denote the model's fixed hyperparameters, the joint posterior distribution of all unknown parameters up to proportionality is

$$
\mathrm{P}\left(\underline{\boldsymbol{\theta}}, \boldsymbol{X}_{\text {miss }} \mid \boldsymbol{X}_{\text {obs }}, \boldsymbol{Y}, \boldsymbol{\Omega}\right) \propto \mathrm{P}\left(\underline{\boldsymbol{\theta}}, \boldsymbol{X}_{\text {miss }} \mid \boldsymbol{\Omega}\right) \mathrm{P}(\boldsymbol{Y} \mid \underline{\boldsymbol{\theta}}, \boldsymbol{X}, \boldsymbol{\Omega}),
$$

where $\boldsymbol{X}_{\text {miss }}$ denotes the missing covariates, $\boldsymbol{X}_{\text {obs }}$ denotes the observed covariates, and $\boldsymbol{X}=\left\{\boldsymbol{X}_{\text {obs }}, \boldsymbol{X}_{\text {miss }}\right\}$. At each MCMC iteration we impute missing values through categorical draws for covariates whose values are missing at random in some samples. We treat each category as equally likely a priori, and the probabilities for the categories are proportional to the likelihood induced by selecting each of those categories.

To improve mixing, at each MCMC iteration we use multiple steps to update $\beta_{j p}$, $\gamma_{j p}$ and $\iota_{p}$. We update $\beta_{j p}$ conditional on all other parameters including $\gamma_{j p}$ and $\iota_{p}$. We also do a joint update of $\gamma_{j p}$ and $\beta_{j p}$ as follows; We use a method similar to that in Carlin and Chib (1995) and generate the joint proposal of $\left(\gamma_{j p}, \beta_{j p}\right)$ using a linking density,

$$
J\left(\hat{\beta}_{j p} \mid \gamma_{j p}^{0}, \beta_{j p}^{0}\right)= \begin{cases}\operatorname{TruncNorm}\left(0, \sigma_{p}^{2},-\iota_{p} \sigma_{p}, \iota_{p} \sigma_{p}\right) & \text { if } \gamma_{j p}^{0}=0 \\ \beta_{j p}^{0} & \text { if } \gamma_{j p}^{0} \neq 0\end{cases}
$$

where $\beta_{j p}^{0}$ and $\gamma_{j p}^{0}$ are the current values of $\beta_{j p}$ and $\gamma_{j p}$. We then set $\hat{\beta}_{j p}^{\prime}=\hat{\beta}_{j p}+e_{j p}^{\beta}$, where $e_{j p}^{\beta}$ is a perturbation drawn from a normal distribution with mean 0 and fixed variance. The joint proposal $\beta_{j p}^{\star}$ and $\gamma_{j p}^{\star}$ is generated as a function of $\hat{\beta}_{j p}^{\prime}$,

$$
F\left(\beta_{j p}^{\star}, \gamma_{j p}^{\star} \mid \hat{\beta}_{j p}^{\prime}\right)= \begin{cases}\beta_{j p}^{\star}=0, \gamma_{j p}^{\star}=0 & \text { if } \iota_{p} \sigma_{p} \geq\left|\hat{\beta}_{j p}^{\prime}\right| \\ \beta_{j p}^{\star}=\hat{\beta}_{j p}^{\prime}, \gamma_{j p}^{\star}=1 & \text { if } \iota_{p} \sigma_{p}<\hat{\beta}_{j p}^{\prime} \\ \beta_{j p}^{\star}=\hat{\beta}_{j p}^{\prime}, \gamma_{j p}^{\star}=2 & \text { if }-\iota_{p} \sigma_{p}>\hat{\beta}_{j p}^{\prime}\end{cases}
$$

The proposal is accepted with probability $\min \left(\mathrm{m}_{M H}, 1\right)$, with the algorithm's MetropolisHastings acceptance ratio $\mathrm{m}_{M H}$ is given by

$$
\begin{aligned}
\mathrm{m}_{M H} & =\frac{\mathrm{P}\left(\boldsymbol{Y}_{j} \mid \gamma_{j p}^{\star}, \beta_{j p}^{\star}, \cdots\right) \mathrm{P}\left(\gamma_{j p}^{\star}\right) \mathrm{P}\left(\beta_{j p}^{\star} \mid \gamma_{j p}^{\star}\right)}{\mathrm{P}\left(\boldsymbol{Y}_{j} \mid \gamma_{j p}^{0}, \beta_{j p}^{0}, \cdots\right) \mathrm{P}\left(\gamma_{j p}^{0}\right) \mathrm{P}\left(\beta_{j p}^{0} \mid \gamma_{j p}^{0}\right)} \frac{J\left(\hat{\beta}_{j p} \mid \gamma_{j p}^{\star}, \beta_{j p}^{\star}\right) G\left(\hat{\beta}_{j p}^{\prime} \mid \hat{\beta}_{j p}\right) F\left(\beta_{j p}^{0}, \gamma_{j p}^{0} \mid \hat{\beta}_{j p}^{\prime}\right)}{J\left(\hat{\beta}_{j p} \mid \gamma_{j p}^{0}, \beta_{j p}^{0}\right) G\left(\hat{\beta}_{j p}^{\prime} \mid \hat{\beta}_{j p}\right) F\left(\beta_{j p}^{\star}, \gamma_{j p}^{\star} \mid \hat{\beta}_{j p}^{\prime}\right)} \\
& =\frac{\mathrm{P}\left(\boldsymbol{Y}_{j} \mid \gamma_{j p}^{\star}, \beta_{j p}^{\star}, \cdots\right) \mathrm{P}\left(\gamma_{j p}^{\star}\right) \mathrm{P}\left(\beta_{j p}^{\star} \mid \gamma_{j p}^{\star}\right)}{\mathrm{P}\left(\boldsymbol{Y}_{j} \mid \gamma_{j p}^{0}, \beta_{j p}^{0}, \cdots\right) \mathrm{P}\left(\gamma_{j p}^{0}\right) \mathrm{P}\left(\beta_{j p}^{0} \mid \gamma_{j p}^{0}\right)} \frac{J\left(\hat{\beta}_{j p} \mid \gamma_{j p}^{\star}, \beta_{j p}^{\star}\right)}{J\left(\hat{\beta}_{j p} \mid \gamma_{j p}^{0}, \beta_{j p}^{0}\right)}
\end{aligned}
$$

where $G\left(\hat{\beta}_{j p}^{\prime} \mid \hat{\beta}_{j p}\right)$ denotes the Gaussian probability density induced by $e_{j p}^{\beta}$ of going from $\hat{\beta}_{j p}$ to $\hat{\beta}_{j p}^{\prime}$. The simplification in the second line can be seen by noting that $F\left(\beta_{j p}, \gamma_{j p} \mid \hat{\beta}_{j p}^{\prime}\right)$ is degenerate. In addition to doing this update individually for each combination of $j$ and $p$, we do a joint update of $\beta_{j p}$ and $\gamma_{j p}$ across all $j$ as well using the straightforward extension of the same algorithm. Lastly, we do a joint update of $\beta_{j p}$ and $\gamma_{j p}$ using a method similar to the 'swap' step of the add/swap/delete algorithm in Li et al. (2017). In this approach, we first choose some covariate $p_{1}$ at random, and then randomly choose a different covariate $p_{2}$ with $\gamma_{j, p_{2}} \neq \gamma_{j, p_{1}}$. In our proposal, we 
swap $\gamma$ for the two selected covariates so that $\gamma_{j, p_{1}}^{\star}=\gamma_{j, p_{2}}^{0}$ and $\gamma_{j, p_{2}}^{\star}=\gamma_{j, p_{1}}^{0}$. Then, conditional on the proposed $\gamma_{j, p_{1}}^{\star}$ and $\gamma_{j, p_{2}}^{\star}$, we generate $\beta_{j, p_{1}}^{\star}$ and $\beta_{j, p_{2}}^{\star}$ from their respective priors. We accept the proposal $\left(\gamma_{j, p_{1}}^{\star}, \beta_{j, p_{1}}^{\star}\right)$ and $\left(\gamma_{j, p_{2}}^{\star}, \beta_{j, p_{2}}^{\star}\right)$ with probability $\min \left(\mathrm{m}_{M H}, 1\right)$ where

$$
\begin{aligned}
\mathrm{m}_{M H}= & \frac{\mathrm{P}\left(\boldsymbol{Y}_{j} \mid \gamma_{j, p_{1}}^{\star}, \beta_{j, p_{1}}^{\star}, \gamma_{j, p_{2}}^{\star}, \beta_{j, p_{2}}^{\star}, \cdots\right) \mathrm{P}\left(\gamma_{j, p_{1}}^{\star}\right) p\left(\gamma_{j, p_{2}}^{\star}\right) \mathrm{P}\left(\beta_{j, p_{1}}^{\star} \mid \gamma_{j, p_{1}}^{\star}\right) \mathrm{P}\left(\beta_{j, p_{2}}^{\star} \mid \gamma_{j, p_{2}}^{\star}\right)}{\mathrm{P}\left(\boldsymbol{Y}_{j} \mid \gamma_{j, p_{1}}^{0}, \beta_{j, p_{1}}^{0}, \gamma_{j, p_{2}}^{0}, \beta_{j, p_{2}}^{0}, \cdots\right) \mathrm{P}\left(\gamma_{j, p_{1}}^{0}\right) \mathrm{P}\left(\gamma_{j, p_{2}}^{0}\right) \mathrm{P}\left(\beta_{j, p_{1}}^{0} \mid \gamma_{j, p_{1}}^{0}\right) \mathrm{P}\left(\beta_{j, p_{2}}^{0} \mid \gamma_{j, p_{2}}^{0}\right)} \\
& \times \frac{Q\left(\left\{\left(\gamma_{j, p_{1}}^{\star}, \beta_{j, p_{1}}^{\star}\right),\left(\gamma_{j, p_{2}}^{\star}, \beta_{j, p_{2}}^{\star}\right)\right\} \rightarrow\left\{\left(\gamma_{j, p_{1}}^{0}, \beta_{j, p_{1}}^{0}\right),\left(\gamma_{j, p_{2}}^{0}, \beta_{j, p_{2}}^{0}\right)\right\}\right)}{Q\left(\left\{\left(\gamma_{j, p_{1}}^{0}, \beta_{j, p_{1}}^{0}\right),\left(\gamma_{j, p_{2}}^{0}, \beta_{j, p_{2}}^{0}\right)\right\} \rightarrow\left\{\left(\gamma_{j, p_{1}}^{\star}, \beta_{j, p_{1}}^{\star}\right),\left(\gamma_{j, p_{2}}^{\star}, \beta_{j, p_{2}}^{\star}\right)\right\}\right)} \\
= & \frac{\mathrm{P}\left(\boldsymbol{Y}_{j} \mid \gamma_{j, p_{1}}^{\star}, \beta_{j, p_{1}}^{\star}, \gamma_{j, p_{2}}^{\star}, \beta_{j, p_{2}}^{\star}, \cdots\right) \mathrm{P}\left(\gamma_{j, p_{1}}^{\star}\right) \mathrm{P}\left(\gamma_{j, p_{2}}^{\star}\right)}{\mathrm{P}\left(\boldsymbol{Y}_{j} \mid \gamma_{j, p_{1}}^{0}, \beta_{j, p_{1}}^{0}, \gamma_{j, p_{2}}^{0}, \beta_{j, p_{2}}^{0}, \cdots\right) \mathrm{P}\left(\gamma_{j, p_{1}}^{0}\right) \mathrm{P}\left(\gamma_{j, p_{2}}^{0}\right)}
\end{aligned}
$$

where $Q$ is the proposal distribution. We repeat this swap step 10 times within each MCMC iteration.

The details of the remaining MCMC simulation steps are described below.

1. $s_{j}, h, \kappa^{2}$ (parameters related to overdispersion parameters): We perform individual metropolis udpates for the over-dispersion parameters $\tilde{s}_{j}=\log \left(s_{j}\right)$. The updates for $h$ and $\kappa^{2}$ are conjugate conditional on $\tilde{s}_{j}$. These updates are the standard Gibbs steps of a normal-normal model with known variance and normal-inverse-gamma with known mean.

- $s_{j}$

$$
\begin{aligned}
\mathrm{P}\left(\tilde{s}_{j} \mid-\right) & \propto \mathrm{N}\left(\tilde{s}_{j} \mid h, \kappa^{2}\right) \\
& \times \prod_{t} \prod_{k=1}^{K_{i}} \frac{\Gamma\left(y_{t k j}+s_{j}^{-1}\right)}{y_{t k j} ! \Gamma\left(s_{j}^{-1}\right)}\left(\frac{\mu_{t k j} s_{j}}{1+\mu_{t k j} s_{j}}\right)^{y_{t k j}}\left(\frac{1}{1+\mu_{t k j} s_{j}}\right)^{s_{j}^{-1}},
\end{aligned}
$$

where '-' represents all other parameters and data. Update via random walk Metropolis-Hastings.

- $h$

$$
h \mid-\sim \mathrm{N}\left(\frac{\frac{1}{b_{h}^{2}} a_{h}+\frac{J}{\kappa^{2}} \bar{s}}{\frac{1}{b_{h}^{2}}+\frac{J}{\kappa^{2}}},\left(\frac{1}{b_{h}^{2}}+\frac{J}{\kappa^{2}}\right)^{-1}\right)
$$

where $\bar{s}=\sum_{j=1}^{J} \tilde{s}_{j}$.

- $\kappa^{2}$

$$
\kappa^{2} \mid-\sim \mathrm{IG}\left(a_{\kappa}+\frac{J}{2}, b_{\kappa}+\frac{1}{2} \sum_{j=1}^{J}\left(\tilde{s}_{j}-h\right)^{2}\right)
$$

2. $\boldsymbol{\psi}^{r}, \boldsymbol{w}^{r}, \boldsymbol{\eta}^{r}$ (parameters related to library size adjustment): 
- $\psi^{r}$

$$
\mathrm{P}\left(\boldsymbol{\psi}^{r} \mid-\right) \propto \prod_{\ell=1}^{L^{r}}\left(\psi_{\ell}^{r}\right)^{a_{\ell}^{\psi^{r}}+d_{\ell}^{r}-1}
$$

Draw from Dirichlet distribution with concentration parameters $a_{\ell}^{\psi^{r}}+d_{\ell}^{r}$.

- $w_{\ell}^{r}$

$$
\begin{aligned}
\mathrm{P}\left(w_{\ell}^{r} \mid-\right) \propto & \prod_{t, k \mid c_{t k}^{r}=\ell}\left(w_{\ell}^{r}\right)^{\lambda_{t k}^{r}}\left(1-w_{\ell}^{r}\right)^{1-\lambda_{t k}^{r}} \prod_{t, k \mid c_{t k}^{r}=\ell \text { and } \lambda_{t k}^{r}=0} \mathrm{~N}\left(r_{t k} \mid \frac{v_{r}-w_{\ell}^{r} \eta_{\ell}^{r}}{1-w_{\ell}^{r}}, u_{r}^{2}\right) \\
& \times\left(w_{\ell}^{r}\right)^{a_{w}^{r}-1}\left(1-w_{\ell}^{r}\right)^{b_{w}^{r}-1} \\
\propto & \left(w_{\ell}^{r}\right)^{a_{w}^{r}+\left[\sum_{t k \mid c_{t k}^{r}=\ell} \lambda_{t k}^{r}\right]-1}\left(1-w_{\ell}^{r}\right)^{b_{w}^{r}+\left[\sum_{t k \mid c_{t k}^{r}=\ell}\left(1-\lambda_{t k}^{r}\right)\right]-1} \\
& \times \prod_{t, k \mid c_{t k}^{r}=\ell \text { and } \lambda_{t k}^{r}=0} \mathrm{~N}\left(r_{t k} \mid \frac{v_{r}-w_{\ell}^{r} \eta_{\ell}^{r}}{1-w_{\ell}^{r}}, u_{r}^{2}\right)
\end{aligned}
$$

Update via random walk Metropolis-Hastings.

- $\eta_{\ell}^{r}$

$$
\eta_{\ell}^{r} \mid-\sim \mathrm{N}\left(\frac{\rho_{\ell}^{r_{1}} m_{\ell}^{r_{1}}+\rho_{\ell}^{r_{2}} m_{\ell}^{r_{2}}+\rho^{r_{3}} m^{r_{3}}}{\rho_{\ell}^{r_{1}}+\rho_{\ell}^{r_{2}}+\rho^{r_{3}}},\left(\rho_{\ell}^{r_{1}}+\rho_{\ell}^{r_{2}}+\rho^{r_{3}}\right)^{-1}\right)
$$

where

$$
\rho_{\ell}^{r_{1}}=\frac{1}{u_{r}^{2}}\left|A_{\ell}^{r}\right|, \quad \rho_{\ell}^{r_{2}}=\frac{1}{u_{r}^{2}}\left(\frac{w_{\ell}^{r}}{1-w_{\ell}^{r}}\right)^{2}\left|B_{\ell}^{r}\right|, \quad \rho^{r_{3}}=\frac{1}{b_{\eta^{r}}^{2}}
$$

and

$$
m_{\ell}^{r_{1}}=\frac{1}{\left|A_{\ell}^{r}\right|} \sum_{A_{\ell}^{r}} r_{t k}, \quad m_{\ell}^{r_{2}}=\frac{1}{\left|B_{\ell}^{r}\right|} \sum_{B_{\ell}^{r}} \frac{v_{r}-\left(1-w_{\ell}^{r}\right) r_{t k}}{w_{\ell}^{r}}, \quad m^{r_{3}}=v_{r},
$$

with $A_{\ell}^{r}=\left\{t, k \mid c_{t k}^{r}=\ell\right.$ and $\left.\lambda_{t k}^{r}=1\right\}$; and $\left|A_{\ell}^{r}\right|$ is the cardinality of this set, $\left|A_{\ell}^{r}\right|=\sum_{t, k} \mathbb{I}\left(c_{t k}^{r}=\ell\right) \mathbb{I}\left(\lambda_{t k}^{r}=1\right)$. Similarly, $B_{\ell}^{r}=\left\{t, k \mid c_{t k}^{r}=\ell\right.$ and $\left.\lambda_{t k}^{r}=0\right\} ;$ and $\left|B_{\ell}^{r}\right|$ is the cardinality of this set, $\left|B_{\ell}^{r}\right|=\sum_{t, k} \mathbb{I}\left(c_{t k}^{r}=\ell\right) \mathbb{I}\left(\lambda_{t k}^{r}=0\right)$.

- $c_{t k}^{r}$

$$
p\left(c_{t k}^{r}=\ell \mid-\right) \propto \psi_{\ell}^{r}\left[w_{\ell}^{r} \mathrm{~N}\left(r_{t k} \mid \eta_{\ell}^{r}, u_{r}^{2}\right)+\left(1-w_{\ell}^{r}\right) \mathrm{N}\left(r_{t k} \mid \frac{v_{r}-w_{\ell}^{r} \eta_{\ell}^{r}}{1-w_{\ell}^{r}}, u_{r}^{2}\right)\right]
$$

Update by drawing from the multinomial distribution with probabilities $p\left(c_{t k}^{r}=\ell \mid-\right)$. 
- $\lambda_{t k}^{r}$

$$
\begin{aligned}
& \mathrm{P}\left(\lambda_{t k}^{r}=1 \mid-\right) \propto w_{c_{t k}} \mathrm{~N}\left(r_{t k} \mid \eta_{c_{t k}^{r}, u_{r}^{2}}\right) \\
& \mathrm{P}\left(\lambda_{t k}^{r}=0 \mid-\right) \propto\left(1-w_{c_{t k}}\right) \mathrm{N}\left(r_{t k} \mid \frac{v_{r}-w_{c_{t k}^{r}}^{r} \eta_{c_{t k}^{r}}^{r}}{1-w_{c_{t k}^{r}}^{r}}, u_{r}^{2}\right)
\end{aligned}
$$

Update by drawing from the Bernoulli distribution with probability $\mathrm{P}\left(\lambda_{t k}^{r}=1 \mid-\right)$.

- $r_{t k}$

$$
\begin{aligned}
\mathrm{P}\left(r_{t k} \mid c_{t k}=\ell,-\right) & \propto \mathrm{N}\left(r_{t k} \mid \eta_{\ell}^{r}, u_{r}^{2}\right)^{\lambda_{t k}^{r}} \mathrm{~N}\left(r_{t k} \mid \frac{v_{r}-w_{\ell}^{r} \eta_{\ell}^{r}}{1-w_{\ell}^{r}}, u_{r}^{2}\right)^{1-\lambda_{t k}^{r}} \\
& \times \prod_{j=1}^{J}\left(\frac{\mu_{t k j} s_{j}}{1+\mu_{t k j} s_{j}}\right)^{y_{t k j}}\left(\frac{1}{1+\mu_{t k j} s_{j}}\right)^{s_{j}^{-1}}
\end{aligned}
$$

Update via random walk Metropolis-Hastings.

3. $\boldsymbol{\psi}^{\alpha}, \boldsymbol{w}^{\alpha}, \boldsymbol{\eta}^{\alpha}$ (OTU baseline abundance parameters)

- $\psi^{\alpha}$

$$
\mathrm{P}\left(\boldsymbol{\psi}^{\alpha} \mid-\right) \propto \prod_{\ell=1}^{L^{\alpha}}\left(\psi_{\ell}^{\alpha}\right)^{a_{\ell}^{\alpha}+d_{\ell}^{\alpha}-1}
$$

Draw from the Dirichlet distribution with concentration parameters $a_{\ell}^{\alpha}+d_{\ell}^{\alpha}$.

- $w_{\ell}^{\alpha}$

$$
\begin{aligned}
\mathrm{P}\left(w_{\ell}^{\alpha} \mid-\right) \propto & \prod_{j \mid c_{j}^{\alpha}=\ell}\left(w_{\ell}^{\alpha}\right)^{\lambda_{j}^{\alpha}}\left(1-w_{\ell}^{\alpha}\right)^{1-\lambda_{j}^{\alpha}} \prod_{j \mid c_{j}^{\alpha}=\ell} \text { and } \lambda_{j}^{\alpha}=0 \\
& \times\left(w_{\ell}^{\alpha}\right)^{a_{w}^{\alpha}-1}\left(1-w_{\ell}^{\alpha}\right)^{b_{w}^{\alpha}-1} \\
\propto & \left(w_{\ell}^{\alpha}\right)^{a_{w}^{\alpha}+\left[\sum_{j \mid c_{j}^{\alpha}=\ell} \lambda_{j}^{\alpha}\right]-1}\left(1-w_{\alpha}-w_{\ell}^{\alpha} \eta_{\ell}^{\alpha}\right. \\
1-w_{\ell}^{\alpha} & b_{w}^{\alpha}+\left[\sum_{j \mid c_{j}^{\alpha}=\ell}\left(1-\lambda_{j}^{\alpha}\right)\right]-1 \\
& \times \prod_{j \mid c_{j}^{\alpha}=\ell} \text { and } \lambda_{j}^{\alpha}=0 \\
& \mathrm{~N}\left(\alpha_{0 j} \mid \frac{v_{\alpha}-w_{\ell}^{\alpha} \eta_{\ell}^{\alpha}}{1-w_{\ell}^{\alpha}}, u_{\alpha}^{2}\right)
\end{aligned}
$$

- $\eta_{\ell}^{\alpha}$

$$
\eta_{\ell}^{\alpha} \mid-\sim \mathrm{N}\left(\frac{\rho_{\ell}^{\alpha_{1}} m_{\ell}^{\alpha_{1}}+\rho_{\ell}^{\alpha_{2}} m_{\ell}^{\alpha_{2}}+\rho^{\alpha_{3}} m^{\alpha_{3}}}{\rho_{\ell}^{\alpha_{1}}+\rho_{\ell}^{\alpha_{2}}+\rho^{\alpha_{3}}},\left(\rho_{\ell}^{\alpha_{1}}+\rho_{\ell}^{\alpha_{2}}+\rho^{\alpha_{3}}\right)^{-1}\right),
$$

where

$$
\rho_{\ell}^{\alpha_{1}}=\frac{1}{u_{\alpha}^{2}}\left|A_{\ell}^{\alpha}\right|, \quad \rho_{\ell}^{\alpha_{2}}=\frac{1}{u_{\alpha}^{2}}\left(\frac{w_{\ell}^{\alpha}}{1-w_{\ell}^{\alpha}}\right)^{2}\left|B_{\ell}^{\alpha}\right|, \quad \rho^{\alpha_{3}}=\frac{1}{b_{\eta^{\alpha}}^{2}}
$$


and

$$
m_{\ell}^{\alpha_{1}}=\frac{1}{\left|A_{\ell}^{\alpha}\right|} \sum_{A_{\ell}^{\alpha}} \alpha_{0 j}, \quad m_{\ell}^{\alpha_{2}}=\frac{1}{\left|B_{\ell}^{\alpha}\right|} \sum_{B_{\ell}^{\alpha}} \frac{v_{\alpha}-\left(1-w_{\ell}^{\alpha}\right) \alpha_{0 j}}{w_{\ell}^{\alpha}}, \quad m^{\alpha_{3}}=v_{\alpha},
$$

with $A_{\ell}^{\alpha}=\left\{j \mid c_{j}^{\alpha}=\ell\right.$ and $\left.\lambda_{j}^{\alpha}=1\right\}$; and $\left|A_{\ell}^{\alpha}\right|$ is the cardinality of this set, $\left|A_{\ell}^{\alpha}\right|=\sum_{j=1}^{J} \mathbb{I}\left(c_{j}^{\alpha}=\ell\right) \mathbb{I}\left(\lambda_{j}^{\alpha}=1\right)$. Similarly, $B_{\ell}^{\alpha}=\left\{j \mid c_{j}^{\alpha}=\ell\right.$ and $\left.\lambda_{j}^{\alpha}=0\right\} ;$ and $\left|B_{\ell}^{\alpha}\right|$ is the cardinality of this set, $\left|B_{\ell}^{\alpha}\right|=\sum_{j=1}^{J} \mathbb{I}\left(c_{j}^{\alpha}=\ell\right) \mathbb{I}\left(\lambda_{j}^{\alpha}=0\right)$.

- $c_{j}^{\alpha}$

$$
\mathrm{P}\left(c_{j}^{\alpha}=\ell \mid-\right) \propto \psi_{\ell}^{\alpha}\left[w_{\ell}^{\alpha} \mathrm{N}\left(\alpha_{0 j} \mid \eta_{\ell}^{\alpha}, u_{\alpha}^{2}\right)+\left(1-w_{\ell}^{\alpha}\right) \mathrm{N}\left(\alpha_{0 j} \mid \frac{v_{\alpha}-w_{\ell}^{\alpha} \eta_{\ell}^{\alpha}}{1-w_{\ell}^{\alpha}}, u_{\alpha}^{2}\right)\right]
$$

Update by drawing from the multinomial distribution with probabilities $p\left(c_{j}^{\alpha}=\ell \mid-\right)$.

- $\lambda_{j}^{\alpha}$

$$
\begin{aligned}
& \mathrm{P}\left(\lambda_{j}^{\alpha}=1 \mid c_{j}^{\alpha}=\ell,-\right) \propto w_{\ell}^{\alpha} \mathrm{N}\left(\alpha_{0 j} \mid \eta_{\ell}^{\alpha}, u_{\alpha}^{2}\right) \\
& \mathrm{P}\left(\lambda_{j}^{\alpha}=0 \mid c_{j}^{\alpha}=\ell,-\right) \propto\left(1-w_{\ell}^{\alpha}\right) \mathrm{N}\left(\alpha_{0 j} \mid \frac{v_{\alpha}-w_{\ell}^{\alpha} \eta_{\ell}^{\alpha}}{1-w_{\ell}^{\alpha}}, u_{\alpha}^{2}\right)
\end{aligned}
$$

Update by drawing from the Bernoulli distribution with probability $\mathrm{P}\left(\lambda_{j}^{\alpha}=\right.$ $1 \mid-)$.

- $\alpha_{0 j}$

$$
\begin{aligned}
\mathrm{P}\left(\alpha_{0 j} \mid c_{j}^{\alpha}=\ell,-\right) & \propto \mathrm{N}\left(\alpha_{0 j} \mid \eta_{\ell}^{\alpha}, u_{\alpha}^{2}\right)^{\lambda_{j}^{\alpha}} \mathrm{N}\left(\alpha_{0 j} \mid \frac{v_{\alpha}-w_{\ell}^{\alpha} \eta_{\ell}^{\alpha}}{1-w_{\ell}^{\alpha}}, u_{\alpha}^{2}\right)^{1-\lambda_{j}^{\alpha}} \\
& \times \prod_{t} \prod_{k=1}^{K_{i}}\left(\frac{\mu_{t k j} s_{j}}{1+\mu_{t k j} s_{j}}\right)^{y_{t k j}}\left(\frac{1}{1+\mu_{t k j} s_{j}}\right)^{s_{j}^{-1}}
\end{aligned}
$$

Update via random walk Metropolis-Hastings.

4. $\theta_{m j}$ and $\tau_{j}^{2}$ (parameters for process convolution)

- $\theta_{m j}$

$$
\mathrm{P}\left(\theta_{m j} \mid-\right) \propto \mathrm{N}\left(\theta_{m j} \mid 0, \tau_{j}^{2}\right) \prod_{t} \prod_{k=1}^{K_{i}}\left(\frac{\mu_{t k j} s_{j}}{1+\mu_{t k j} s_{j}}\right)^{y_{t k j}}\left(\frac{1}{1+\mu_{t k j} s_{j}}\right)^{s_{j}^{-1}}
$$

Update via random walk Metropolis-Hastings.

- $\tau_{j}^{2}$

$$
\tau_{j}^{2} \mid-\sim \mathrm{IG}\left(a_{\tau}+\frac{M}{2}, b_{\tau}+\frac{1}{2} \sum_{m=1}^{M} \theta_{m j}^{2}\right)
$$


5. $\pi_{p 0}^{\star}, \pi_{p 1}, \beta_{j p}, \gamma_{j p}, \iota_{p}$ and $\sigma_{j}^{2}$ (parameters related to covariate effects)

- $\pi_{p 0}^{\star}$

$$
\pi_{p 0}^{\star} \mid-\sim \operatorname{Be}\left(a_{\pi 0}+\sum_{j=1}^{J} \mathbb{I}\left(\gamma_{j p}=0\right), b_{\pi 0}+\sum_{j=1}^{J} \mathbb{I}\left(\gamma_{j p} \neq 0\right)\right)
$$

- $\pi_{p 1}$

$$
\pi_{p 1} \mid-\sim \operatorname{Be}\left(a_{\pi 1}+\sum_{j=1}^{J} \mathbb{I}\left(\gamma_{j p}=1\right), b_{\pi 1}+\sum_{j=1}^{J} \mathbb{I}\left(\gamma_{j p}=2\right)\right)
$$

- $\sigma_{p}^{2}$

$$
\begin{aligned}
\mathrm{P}\left(\sigma_{p}^{2} \mid-\right) & \propto\left(\sigma_{p}^{2}\right)^{-a_{\sigma}-1} \exp \left(-\frac{b_{\sigma}}{\sigma_{p}^{2}}\right) \\
& \times \prod_{j=1}^{J}\left[\mathrm{~N}\left(\beta_{j p} \mid 0, \sigma_{p}^{2}\right)\right]^{\mathbb{I}\left(\gamma_{j p}=2\right)}\left[\mathrm{N}\left(\beta_{j p} \mid 0, \sigma_{p}^{2}\right)\right]^{\mathbb{I}\left(\gamma_{j p}=1\right)} \\
& \times \mathbb{I}\left(\sigma_{p}^{2}<U_{\sigma_{p}^{2}}\right)
\end{aligned}
$$

where $U_{\sigma_{p}^{2}}$ is an upper bound given by $\min \left\{\left(\beta_{j p} / \iota_{p}\right)^{2}\right.$, for $j$ with $\left.\gamma_{j p} \neq 0\right\}$.

Update by drawing $\sigma_{p}^{2}$ from IG $\left(a_{\sigma}+\sum_{j=1}^{J} I\left(\gamma_{j p} \neq 0\right) / 2, \quad b_{\sigma}+\sum_{j=1}^{J} \beta_{j p}^{2} / 2\right)$, but truncated at $U_{\sigma_{p}^{2}}$.

- $\iota_{p}$

$$
\begin{aligned}
\mathrm{P}\left(\iota_{p} \mid-\right) & \propto \iota_{p}^{a_{\iota}-1} \exp \left(-b_{\iota} \iota_{p}\right) \\
& \times\left(\frac{1}{\Phi\left(-\iota_{p}\right)}\right)^{\sum_{j=1}^{J} \mathbb{I}\left(\gamma_{j p}=2\right)} \times\left(\frac{1}{1-\Phi\left(\iota_{p}\right)}\right)^{\sum_{j=1}^{J} \mathbb{I}\left(\gamma_{j p}=1\right)} \times \mathbb{I}\left(\iota_{p}>U_{\iota_{p}}\right),
\end{aligned}
$$

where $U_{\iota_{p}}=\min \left\{\left|\beta_{j p}\right| / \sigma_{p}\right.$, for $j$ with $\left.\gamma_{j p} \neq 0\right\}$. Update via random walk Metropolis-Hastings.

- $\beta_{j p}$

We do not update $\beta_{j p}$ if $\gamma_{j p}=0$. For $\beta_{j p}$ with $\gamma_{j p}=1$ :

$\mathrm{P}\left(\beta_{j p} \mid \gamma_{j p}=1,-\right) \propto \frac{\phi\left(\beta_{j p} \mid 0, \sigma_{p}^{2}\right)}{1-\Phi\left(\iota_{p}\right)} \mathbb{I}\left(\frac{\beta_{j p}}{\sigma_{p}}>\iota_{p}\right) \prod_{i=1}^{n} \prod_{k=1}^{K_{i}}\left(\frac{\mu_{t k j} s_{j}}{1+\mu_{t k j} s_{j}}\right)^{y_{t k j}}\left(\frac{1}{1+\mu_{t k j} s_{j}}\right)^{s_{j}^{-1}}$

Use a Metropolis-Hastings algorithm to update using proposal TruncNorm $\left(\beta_{j p}^{\star},\left(\sigma^{2}\right)^{\prime}, \iota_{p} \sigma_{p}, \infty\right)$, where $\left(\sigma^{2}\right)^{\prime}$ is a fixed proposal variance. For $\beta_{j p}$ with $\gamma_{j p}=2$ :

$$
\mathrm{P}\left(\beta_{j p} \mid \gamma_{j p}=2,-\right) \propto \frac{\phi\left(\beta_{j p} \mid 0, \sigma_{p}^{2}\right)}{\Phi\left(-\iota_{p}\right)} \mathbb{I}\left(\frac{\beta_{j p}}{\sigma_{p}}<-\iota_{p}\right) \prod_{i=1}^{n} \prod_{k=1}^{K_{i}}\left(\frac{\mu_{t k j} s_{j}}{1+\mu_{t k j} s_{j}}\right)^{y_{t k j}}\left(\frac{1}{1+\mu_{t k j} s_{j}}\right)^{s_{j}^{-1}}
$$




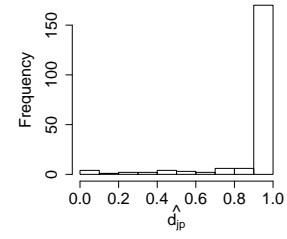

(a) $x_{1}$

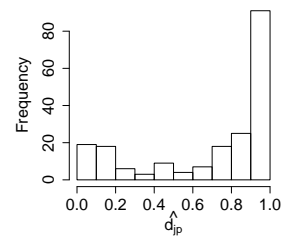

(d) $x_{4}$

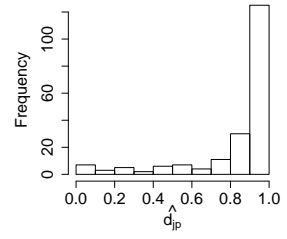

(b) $x_{2}$

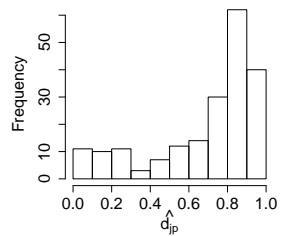

(e) $x_{5}$

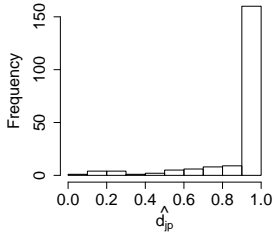

(c) $x_{3}$

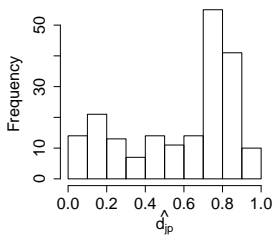

(f) $x_{6}$

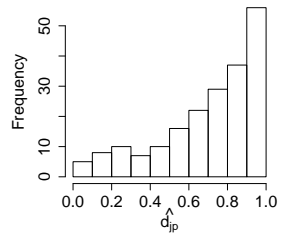

(g) $x_{7}$

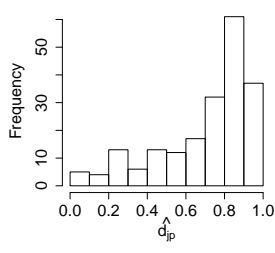

(h) $x_{8}$

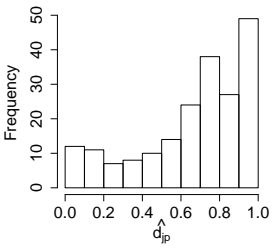

(i) $x_{9}$

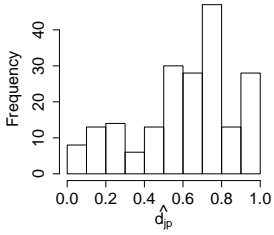

(j) $x_{10}$

Figure 1: [Simulation 1] Histograms of $\hat{d}_{j p}=\hat{\mathrm{P}}\left(\gamma_{j p}=\gamma_{j p}^{\mathrm{TR}} \mid \boldsymbol{Y}\right)$.

Do a metropolis update using proposal TruncNorm $\left(\beta_{j p}^{\star},\left(\sigma^{2}\right)^{\prime},-\infty,-\iota_{p} \sigma_{p}\right)$, where $\left(\sigma^{2}\right)^{\prime}$ is a fixed proposal variance.

\section{Additional Results for Simulation 1}

In this section we present additional results from Simulation 1 in $\S 3$ of the main text. Figure 1 has histograms of posterior estimates $\hat{d}_{j p}=\hat{\mathrm{P}}\left(\gamma_{j p}=\gamma_{j p}^{\mathrm{TR}} \mid \boldsymbol{Y}\right)$, the probabilities that $\beta_{j p}$ is correctly selected with its true direction. Figure 2 compares the posterior mean estimates $\hat{\beta}_{j p}$ to their true values $\beta_{j p}^{\mathrm{TR}}$ for all covariates. Note that covariates $x_{1}-x_{3}$ are continuous covariates and $x_{4}-x_{10}$ are binary indicators to represent different concentration levels of Alexandrium (Ax) and domoic acid (DA). Figure 3(a) compares posterior estimates $\hat{g}_{t k j}$ of baseline mean counts to their true values. The difference of 


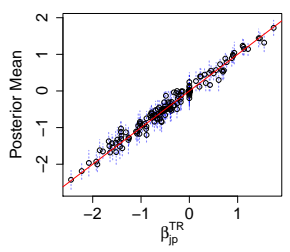

(a) $x_{1}$

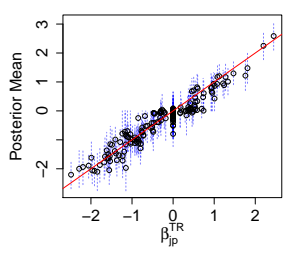

(d) $x_{4}$

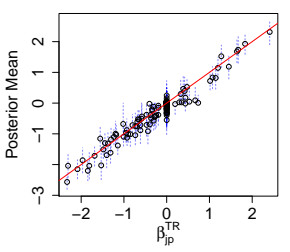

(b) $x_{2}$

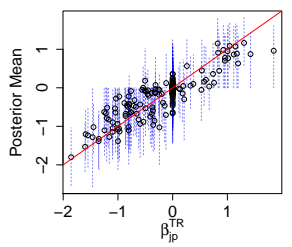

(e) $x_{5}$

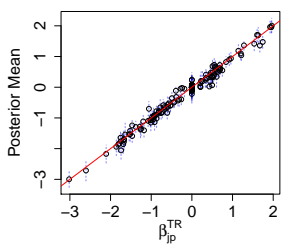

(c) $x_{3}$

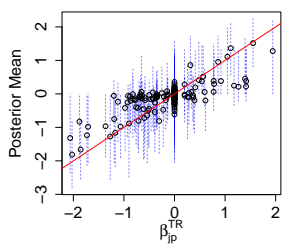

(f) $x_{6}$

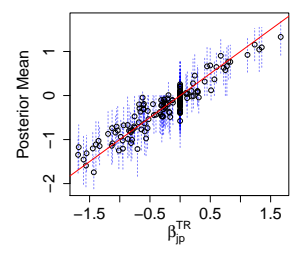

(g) $x_{7}$

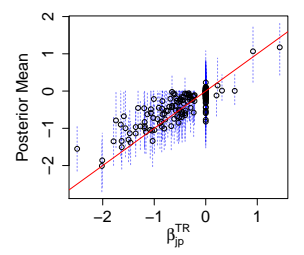

(h) $x_{8}$

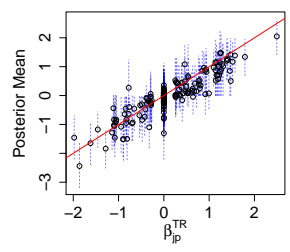

(i) $x_{9}$

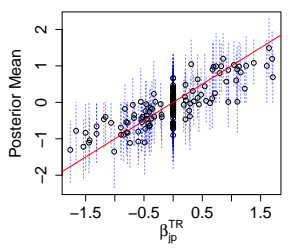

(j) $x_{10}$

Figure 2: [Simulation 1] Posterior means of the regression coefficients $\hat{\beta}_{j p}$ versus their true values $\beta_{j p}^{\mathrm{TR}}$. The dashed blue lines show $95 \%$ posterior credible intervals, and the solid red lines are 45 degree reference lines.

$g_{t k j}^{\mathrm{TR}}-\hat{g}_{t k j}$ is distributed tightly around zero, indicating the baseline counts are well estimated. Figure 3(b) and (c) compare posterior estimates $\hat{r}_{t k}$ and $\hat{\alpha}_{0 j}$ of library size adjustment factors $r_{t k}$ and OTU specific baseline abundance $\alpha_{0 j}$ to their true values, respectively. We observe vertical shifts from the true values in opposite directions; that is, $r_{t k} \mathrm{~s}$ are underestimated for all OTUs and $\alpha_{0 j}$ are overestimated due to the lack of identifiability in the construction of $g_{t k j}$. Figure $3(\mathrm{~d})$-(f) show posterior estimates $\hat{\alpha}_{t j}$ of the temporal structure. In the figure, we plot $\alpha_{0 j}^{\mathrm{TR}}+\hat{\alpha}_{t j}$ over $t$ for some selected OTUs. For easy comparison to the truth, $\alpha_{0 j}^{\mathrm{TR}}$ is used instead of posterior estimates of $\alpha_{0 j}$. The red line in the figure represents the simulation truth. Sample time points are shown by open black circles, and the posterior mean is shown by a black line with pointwise $95 \%$ credible intervals (blue dashed lines). Overall, the model does a good job of capturing the temporal dependence introduced by the sampling procedure. 


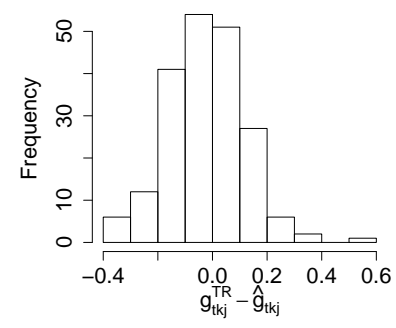

(a) $\left(g_{t k j}^{\mathrm{TR}}-\hat{g}_{t k j}\right)$

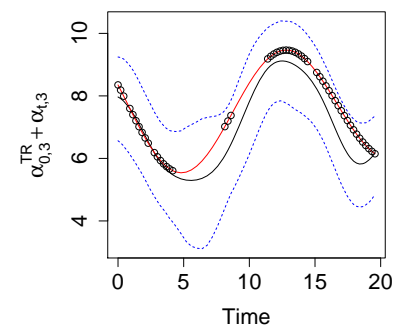

(d) $\hat{\alpha}_{t j}$ with $j=3$

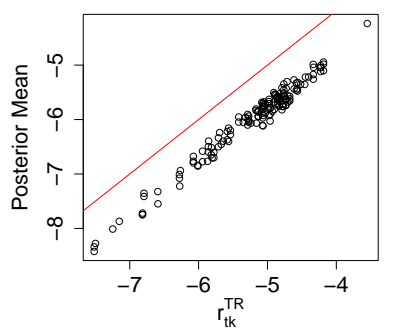

(b) $\hat{r}_{t j}$ vs $r_{t j}^{\mathrm{TR}}$

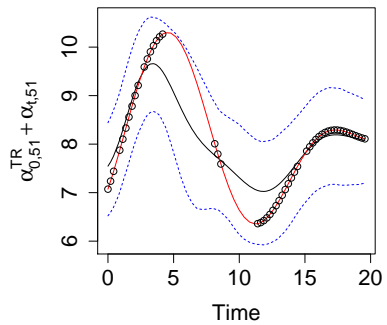

(e) $\hat{\alpha}_{t j}$ with $j=51$

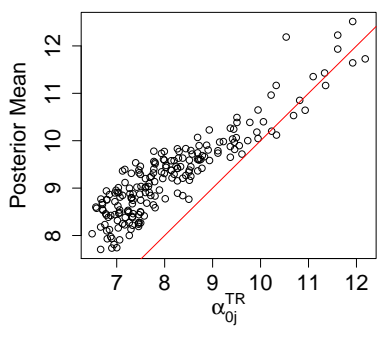

(c) $\hat{\alpha}_{0 j} \operatorname{vs} \alpha_{0 j}^{\mathrm{TR}}$

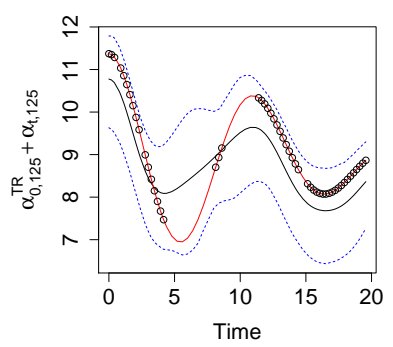

(f) $\hat{\alpha}_{t j}$ with $j=125$

Figure 3: [Simulation 1] Panel (a): Histogram of the differences between the posterior means of the baseline mean counts $\hat{g}_{t k j}$ and their true values $g_{t k j}^{\mathrm{TR}}$. Panel (b): Posterior means of the sample scale factors $\hat{r}_{t k}$ versus their true values $r_{t k}^{\mathrm{TR}}$. Panel (c): Posterior means of the OTU-specific baseline abundance $\hat{\alpha}_{0 j}$ versus their true values $\alpha_{0 j}^{\mathrm{TR}}$. Panels (d) through (f): Posterior means $\hat{\alpha}_{t j}$ of $\alpha_{t j}$ (black, solid) compared to the simulation truth (red, solid) for some selected OTUs with 95\% credible intervals (blue, dotted).

We examined the sensitivity of the estimation of $\beta_{j p}$ and $\gamma_{j p}$ to the prior specification of $\iota_{p}$ and $\sigma_{p}$ for ANLP-SB. Recall that $\iota_{p} \stackrel{i i d}{\sim} \operatorname{Gamma}\left(a_{\iota}, b_{\iota}\right)$ and $\sigma_{p}^{2} \stackrel{i i d}{\sim} \operatorname{IG}\left(a_{\sigma}, b_{\sigma}\right)$ are assumed. Six different specifications of $\left(a_{\iota}, b_{\iota}, a_{\sigma}, b_{\sigma}\right)$ are given in Table 1 , including the specification used in $\S 3$ of the main text to facilitate easy comparison. Results are illustrated in Figure 4 for $x_{1}$ and $x_{3}$, the same covariates in Figure 3 in the main text. The figures show that the model is not overly sensitive to the prior specification of $\iota_{p}$ and $\sigma_{p}^{2}$ within a reasonable range. We found that overdispersed priors for $\iota_{p}$ may lead to poor convergence and/or inference (results are not shown). We also examined the sensitivity of the estimation of $g_{t k j}$ by varying the number of knot points $M$ and the values of the prespecified mean constraints $v_{r}$ and $v_{\alpha}$. We tried $M=50$ and $M=90$ and compared the results to those with $M=70$. We found relatively little impact on the posterior inference. The model is more sensitive to changes in the range parameter, as the range parameter can be chosen in such a way that the temporal dependence between sample points is too strong. We also specified $v_{r}$ and $v_{\alpha}$ by \pm 2 and $\mp 2$ to the values 


\begin{tabular}{l|llll}
\hline & $a_{\sigma}$ & $b_{\sigma}$ & $a_{\iota}$ & $b_{\iota}$ \\
\hline Case 1 & 1 & 1 & 2.5 & 10 \\
Case 2 & 0.1 & 0.1 & 2.5 & 10 \\
Case 3 & 1 & 1 & 1 & 10 \\
Case 4 & 0.1 & 0.1 & 1 & 10 \\
Case 5 & 1 & 1 & 5 & 20 \\
Case 6 & 0.1 & 0.1 & 5 & 20 \\
\hline
\end{tabular}

Table 1: Prior specifications for $\iota_{p}$ and $\sigma_{p}^{2} . \iota_{p} \stackrel{i i d}{\sim} \operatorname{Gamma}\left(a_{\iota}, b_{\iota}\right)$ and $\sigma_{p}^{2} \stackrel{i i d}{\sim} \operatorname{IG}\left(a_{\sigma}, b_{\sigma}\right)$ are assumed.

\begin{tabular}{rccccc}
\hline Model & MCC & ACC & AUC & Brier-Score & $\mathrm{F}_{1}$ \\
\hline ANLP-SB & $\mathbf{0 . 6 1 3}(0.044)$ & $\mathbf{0 . 8 0 2}(0.021)$ & $\mathbf{0 . 8 8 6}(0.018)$ & $\mathbf{0 . 2 8 6}(0.031)$ & $\mathbf{0 . 7 8 8}(0.021)$ \\
ALP-SB & $0.294(0.042)$ & $0.606(0.029)$ & $0.781(0.025)$ & $0.547(0.047)$ & $0.710(0.026)$ \\
SLP-SB & $0.288(0.039)$ & $0.604(0.028)$ & $0.775(0.023)$ & - & $0.708(0.026)$ \\
BayesReg & $0.530(0.035)$ & $0.741(0.023)$ & $0.799(0.019)$ & - & $0.676(0.024)$ \\
edgeR-L & $-0.003(0.031)$ & $0.498(0.016)$ & $0.499(0.020)$ & - & $0.442(0.027)$ \\
edgeR-Q & $0.002(0.031)$ & $0.501(0.016)$ & $0.502(0.019)$ & - & $0.474(0.025)$ \\
BhGLM & $0.231(0.043)$ & $0.602(0.024)$ & $0.635(0.026)$ & - & $0.491(0.032)$ \\
\hline
\end{tabular}

(a) Variable Selection

\begin{tabular}{|c|c|c|c|c|c|}
\hline \multirow{2}{*}{ Model } & \multicolumn{3}{|c|}{ RMSE } & \multirow{2}{*}{ DIC } & \multirow{2}{*}{ LPML } \\
\hline & $\beta_{j p}$ & $\pi_{p 0}^{\star}$ & $g_{t k j}$ & & \\
\hline ANLP-SB & $\mathbf{0 . 2 8 0}(0.023)$ & $\mathbf{0 . 0 8 8}(0.025)$ & $0.322(0.030)$ & $\mathbf{2 4 1 , 9 4 7}(6,214)$ & -4.036 (0.104) \\
\hline ALP-SB & $0.297(0.017)$ & $0.282(0.031)$ & $0.339(0.015)$ & $242,030(6,210)$ & $-4.038(0.104)$ \\
\hline SLP-SB & $0.303(0.016)$ & $0.281(0.031)$ & $0.350(0.018)$ & $242,058(6,209)$ & $-4.039(0.104)$ \\
\hline BayesReg & $0.304(0.016)$ & - & $0.355(0.029)$ & $242,177(6,219)$ & $-4.044(0.104)$ \\
\hline edgeR-L & $0.870(0.032)$ & - & - & - & - \\
\hline edgeR-Q & $0.861(0.028)$ & - & - & - & - \\
\hline BhGLM & $0.976(0.063)$ & - & - & - & - \\
\hline
\end{tabular}

(b) Parameter Estimation and Model Fit

Table 2: [Simulation 2: Comparison] Performance metric averages over 100 simulated datasets with standard deviations in parenthesis. The best performances are in bold.

empirically specified as described in the main text. We found that these changes had little impact on the posterior estimates of $g_{t k j}$. Histograms of the differences between the true baseline mean counts $g_{t k j}^{\mathrm{TR}}$ and their posterior estimates $\hat{g}_{t k j}$ under the different simulation conditions are shown in Figure 5.

We diagnosed the convergence of the posterior MCMC simulation using trace plots of the log-likelihood. Figure 6 shows trace plots of the log-likehood based on imputed parameters under the six different hyperparameter specifications in Table 1. The chains converge to similar log-likelihood ranges regardless of the hyperparameter specification, which provides no evidence of poor convergence or poor mixing. Examination of trace plots and autoccorrelation plots for some parameters also show practical evidence of posterior convergence and good mixing (not shown). 


\begin{tabular}{rccccc}
\hline Model & MCC & ACC & AUC & Brier-Score & $\mathrm{F}_{1}$ \\
\hline ANLP-SB & $\mathbf{0 . 4 6 1}(0.038)$ & $\mathbf{0 . 7 3 0}(0.019)$ & $\mathbf{0 . 8 2 6}(0.016)$ & $\mathbf{0 . 3 9 3}(0.027)$ & $\mathbf{0 . 7 3 9}(0.018)$ \\
ALP-SB & $0.247(0.037)$ & $0.587(0.028)$ & $0.743(0.021)$ & $0.609(0.043)$ & $0.698(0.027)$ \\
SLP-SB & $0.240(0.037)$ & $0.584(0.028)$ & $0.736(0.019)$ & - & $0.696(0.027)$ \\
BayesReg & $0.450(0.035)$ & $0.711(0.022)$ & $0.759(0.020)$ & - & $0.652(0.024)$ \\
edgeR-L & $-0.003(0.033)$ & $0.498(0.022)$ & $0.498(0.018)$ & - & $0.328(0.034)$ \\
edgeR-Q & $0.001(0.031)$ & $0.499(0.023)$ & $0.502(0.017)$ & - & $0.282(0.029)$ \\
BhGLM & $0.389(0.064)$ & $0.665(0.029)$ & $0.732(0.038)$ & - & $0.549(0.039)$ \\
\hline
\end{tabular}

(a) Variable Selection

\begin{tabular}{|c|c|c|c|c|c|}
\hline \multirow{2}{*}{ Model } & \multicolumn{3}{|c|}{ RMSE } & \multirow{2}{*}{ DIC } & \multirow{2}{*}{ LPML } \\
\hline & $\beta_{j p}$ & $\pi_{p 0}^{\star}$ & $g_{t k j}$ & & \\
\hline ANLP-SB & $0.393(0.021)$ & $0.102(0.030)$ & $0.716(0.014)$ & $247,071(6,073)$ & $-4.124(0.101)$ \\
\hline ALP-SB & $\mathbf{0 . 3 9 0}(0.019)$ & $0.285(0.031)$ & $0.712(0.012)$ & $247,078(6,070)$ & $-4.124(0.101)$ \\
\hline SLP-SB & $0.393(0.016)$ & $0.285(0.031)$ & $0.703(0.011)$ & $247,089(6,073)$ & $-4.125(0.101)$ \\
\hline BayesReg & $\mathbf{0 . 3 9 0}(0.016)$ & - & $0.707(0.015)$ & $247,742(6,089)$ & $-4.140(0.102)$ \\
\hline edgeR-L & $0.487(0.014)$ & - & - & - & - \\
\hline edgeR-Q & $0.491(0.014)$ & - & - & - & - \\
\hline BhGLM & $0.526(0.062)$ & - & - & - & - \\
\hline
\end{tabular}

Table 3: [Simulation 3: Comparison] Performance metric averages over 100 simulated datasets with standard deviations in parenthesis. The best performances are in bold.

\section{Simulations 2-8}

\subsection{Simulations 2 and 3}

We conducted additional simulations for further examination of the proposed ANLPSB model. We compared ANLP-SB to the five competing models: ALP-SB, SLP-SB, BayesReg, edgeR, and BhGLM. The simulation setup for Simulations 2 and 3 is similar to Simulation 1's setup, including the specification for $\boldsymbol{x}_{t}, r_{t k}^{\mathrm{TR}}$ and $\alpha_{0 j}^{\mathrm{TR}}$. Simulation 2 was setup to investigate the models' performance when the regression coefficients for irrelevant covariates are not exactly zero. In particular, we let $\beta_{j p}^{\text {TR }} \stackrel{\text { indep }}{\sim} \mathrm{N}\left(0,\left(\iota_{p} / 6\right)^{2}\right)$ for covariates with $\gamma_{j p}^{\mathrm{TR}} \neq 0$, giving these covariates negligible but non-zero effects on OTU abundance. Recall that the model assumes $\beta_{j p}^{\mathrm{TR}}=0$ when $\gamma_{j p}^{\mathrm{TR}}=0$. For Simulation 3, we let $\alpha_{t j}^{\mathrm{TR}} \stackrel{\text { indep }}{\sim} \mathrm{N}\left(0,(2 / 3)^{2}\right)$; that is, no temporal dependence in the simulation truth was assumed. Tables 2 and 3 show performance metrics for Simulations 2 and 3 , respectively. In both simulations, ANLP-SB outperforms the competing models, especially with regard to variable selection. ANLP-SB performs notably better than the other Bayesian models in terms of the RMSE of $\pi_{p 0}^{\star}, \mathrm{MCC}, \mathrm{AUC}$, and Brier score. The four Bayesian models have similar performance for parameter estimation based on the RMSE and similar model fit based on DIC and LPML. 


\begin{tabular}{l|lllll}
\hline & $J$ & $P$ & $n$ & $N$ & Run-time \\
\hline Simulation 1 & 200 & 10 & 54 & 150 & 9 \\
Simulation 2 & 200 & 10 & 54 & 150 & 8 \\
Simulation 3 & 200 & 10 & 54 & 150 & 9 \\
Simulation 4 & 400 & 10 & 50 & 100 & 12 \\
Simulation 5 & 400 & 20 & 50 & 100 & 16 \\
Simulation 6 & 400 & 10 & 100 & 200 & 23 \\
Simulation 7 & 400 & 20 & 100 & 200 & 29 \\
Simulation 8 & 200 & 50 & 100 & 200 & 22 \\
\hline
\end{tabular}

Table 4: Simulation setups $(J, P, n, N)$ for Simulations $1-8$. The run-times (in minutes) for 1,000 MCMC iterations are reported in the last column.

\begin{tabular}{rccccc}
\hline Model & MCC & ACC & AUC & Brier-Score & $F_{1}$ \\
\hline ANLP-SB & $\mathbf{0 . 7 0 3}(0.137)$ & $\mathbf{0 . 8 5 3}(0.073)$ & $\mathbf{0 . 9 2 5}(0.062)$ & $\mathbf{0 . 2 5 3}(0.141)$ & $\mathbf{0 . 8 5 1}(0.076)$ \\
ALP-SB & $0.298(0.093)$ & $0.595(0.128)$ & $0.785(0.025)$ & $0.647(0.193)$ & $0.687(0.127)$ \\
SLP-SB & $0.298(0.095)$ & $0.588(0.135)$ & $0.799(0.026)$ & - & $0.687(0.128)$ \\
BayesReg & $0.589(0.078)$ & $0.788(0.059)$ & $0.831(0.027)$ & - & $0.744(0.032)$ \\
edgeR-L & $-0.043(0.098)$ & $0.501(0.087)$ & $0.475(0.062)$ & - & $0.301(0.106)$ \\
edgeR-Q & $-0.038(0.113)$ & $0.501(0.081)$ & $0.477(0.071)$ & - & $0.335(0.109)$ \\
BhGLM & $0.337(0.054)$ & $0.621(0.105)$ & $0.720(0.042)$ & - & $0.386(0.058)$ \\
\hline
\end{tabular}

(a) Variable Selection

\begin{tabular}{|c|c|c|c|c|c|}
\hline \multirow{2}{*}{ Model } & \multicolumn{3}{|c|}{ RMSE } & \multirow{2}{*}{ DIC } & \multirow{2}{*}{ LPML } \\
\hline & $\beta_{j p}$ & $\pi_{p 0}^{\star}$ & $g_{t k j}$ & & \\
\hline ANLP-SB & $0.125(0.028)$ & $0.246(0.130)$ & $\mathbf{0 . 3 6 8}(0.071)$ & $\mathbf{3 5 9 , 1 3 8}(8,220)$ & $\mathbf{- 4 . 5 0 6}(0.103)$ \\
\hline ALP-SB & $0.157(0.020)$ & $0.474(0.113)$ & $0.476(0.099)$ & $359,381(8,188)$ & $-4.512(0.103)$ \\
\hline SLP-SB & $0.176(0.035)$ & $0.462(0.112)$ & $0.633(0.187)$ & $359,515(8,187)$ & $-4.514(0.103)$ \\
\hline BayesReg & $0.217(0.037)$ & - & $0.815(0.214)$ & $361,391(8,196)$ & $-4.545(0.102)$ \\
\hline edgeR-L & $0.341(0.031)$ & - & - & - & - \\
\hline edgeR-Q & $0.313(0.031)$ & - & - & - & - \\
\hline BhGLM & $0.357(0.044)$ & - & - & - & - \\
\hline
\end{tabular}

(b) Parameter Estimation and Model Fit

Table 5: [Simulation 4: Comparison] Results from the simulation study with $N=100$ samples taken at $n=50$ time points with $J=400$ OTUs and $P=10$ covariates.

\subsection{Simulations $4-8$}

We performed Simulations 4-8 to examine the models' performance in higher dimensional settings by using different numbers of OTUs $(J)$, covariates $(P)$, and samples $(N)$. We fixed $K=2$ and used values of $(J, P, N)$ in Table 4 . For all simulations, we let $\boldsymbol{x}_{t} \stackrel{i i d}{\sim} \mathbf{N}(\mathbf{0}, \Sigma)$, where the diagonal of $\boldsymbol{\Sigma}$ was 1, and the off diagonals were $1 / 4$. Similar to Simulations 2-3, we used $r_{t k}^{\prime}$ and $\alpha_{0 j}^{\prime}$ computed from the ocean microbiome data to set $r_{t k}^{\mathrm{TR}}$ and $\alpha_{0 j}^{\mathrm{TR}}$; let $r_{t k}^{\mathrm{TR}}=\log \left(r_{t k}^{\prime \prime}+\epsilon_{t k}^{r}\right)$ and $\alpha_{0 j}^{\mathrm{TR}}=\log \left(\alpha_{0 j}^{\prime \prime}+\epsilon_{j}^{\alpha}\right)$, where $r_{t k}^{\prime \prime}$ and $\alpha_{0 j}^{\prime \prime}$ were random draws from $\left\{r_{t k}^{\prime}\right\}$ and $\left\{\alpha_{0 j}^{\prime}\right\}$, respectively, $\epsilon_{t k}^{r} \stackrel{i i d}{\sim} \mathrm{N}\left(0,10^{-5}\right)$ and $\epsilon_{j}^{\alpha} \stackrel{i i d}{\sim} \mathrm{N}(0,1 / 10)$. 


\begin{tabular}{rccccc}
\hline Model & MCC & ACC & AUC & Brier-Score & $\mathrm{F}_{1}$ \\
\hline ANLP-SB & $\mathbf{0 . 7 4 1}(0.089)$ & $\mathbf{0 . 8 7 2}(0.043)$ & $\mathbf{0 . 9 5 7}(0.034)$ & $\mathbf{0 . 2 0 4}(0.075)$ & $\mathbf{0 . 8 6 0}(0.042)$ \\
ALP-SB & $0.227(0.061)$ & $0.553(0.106)$ & $0.811(0.020)$ & $0.668(0.154)$ & $0.672(0.102)$ \\
SLP-SB & $0.202(0.067)$ & $0.538(0.111)$ & $0.832(0.022)$ & - & $0.667(0.103)$ \\
BayesReg & $0.567(0.052)$ & $0.768(0.051)$ & $0.815(0.018)$ & - & $0.704(0.024)$ \\
edgeR-L & $-0.028(0.060)$ & $0.505(0.069)$ & $0.483(0.037)$ & - & $0.272(0.078)$ \\
edgeR-Q & $-0.003(0.082)$ & $0.514(0.068)$ & $0.496(0.052)$ & - & $0.307(0.085)$ \\
BhGLM & $0.285(0.042)$ & $0.602(0.086)$ & $0.683(0.030)$ & - & $0.331(0.056)$ \\
\hline
\end{tabular}

(a) Variable Selection

\begin{tabular}{|c|c|c|c|c|c|}
\hline \multirow{2}{*}{ Model } & \multicolumn{3}{|c|}{ RMSE } & \multirow{2}{*}{ DIC } & \multirow{2}{*}{ LPML } \\
\hline & $\beta_{j p}$ & $\pi_{p 0}^{\star}$ & $g_{t k j}$ & & \\
\hline ANLP-SB & $\mathbf{0 . 1 4 0}(0.030)$ & $\mathbf{0 . 1 9 4}(0.066)$ & $0.491(0.103)$ & $\mathbf{3 6 6 , 3 4 7}(13,317)$ & $-4.611(0.168)$ \\
\hline ALP-SB & $0.167(0.017)$ & $0.492(0.077)$ & $0.618(0.110)$ & $366,577(13,222)$ & $-4.625(0.167)$ \\
\hline SLP-SB & $0.185(0.025)$ & $0.483(0.077)$ & $0.975(0.260)$ & $366,873(13,318)$ & $-4.629(0.167)$ \\
\hline BayesReg & $0.222(0.024)$ & - & $1.198(0.269)$ & $368,725(13,276)$ & $-4.669(0.167)$ \\
\hline edgeR-L & $0.407(0.031)$ & - & - & - & - \\
\hline edgeR-Q & $0.377(0.033)$ & - & - & - & - \\
\hline BhGLM & $0.439(0.066)$ & - & - & - & - \\
\hline
\end{tabular}

(b) Parameter Estimation and Model Fit

Table 6: [Simulation 5: Comparison] Results from the simulation study with $N=100$ samples taken at $n=50$ time points with $J=400$ OTUs and $P=20$ covariates.

\begin{tabular}{rccccc}
\hline Model & MCC & ACC & AUC & Brier-Score & $\mathrm{F}_{1}$ \\
\hline ANLP-SB & $\mathbf{0 . 7 5 4}(0.148)$ & $\mathbf{0 . 8 7 9}(0.076)$ & $\mathbf{0 . 9 3 0}(0.063)$ & $\mathbf{0 . 2 1 8}(0.150)$ & $\mathbf{0 . 8 7 6}(0.095)$ \\
ALP-SB & $0.378(0.091)$ & $0.643(0.121)$ & $0.837(0.018)$ & $0.581(0.193)$ & $0.717(0.132)$ \\
SLP-SB & $0.388(0.096)$ & $0.640(0.129)$ & $0.852(0.020)$ & - & $0.718(0.134)$ \\
BayesReg & $0.671(0.065)$ & $0.838(0.040)$ & $0.878(0.021)$ & - & $0.818(0.031)$ \\
edgeR-L & $-0.043(0.137)$ & $0.505(0.085)$ & $0.474(0.084)$ & - & $0.392(0.145)$ \\
edgeR-Q & $-0.043(0.146)$ & $0.503(0.078)$ & $0.475(0.090)$ & - & $0.422(0.150)$ \\
BhGLM & $0.467(0.064)$ & $0.697(0.081)$ & $0.788(0.033)$ & - & $0.578(0.041)$ \\
\hline
\end{tabular}

(a) Variable Selection

\begin{tabular}{|c|c|c|c|c|c|}
\hline \multirow{2}{*}{ Model } & \multicolumn{3}{|c|}{ RMSE } & \multirow{2}{*}{ DIC } & \multirow{2}{*}{ LPML } \\
\hline & $\beta_{j p}$ & $\pi_{p 0}^{\star}$ & $g_{t k j}$ & & \\
\hline ANLP-SB & $\mathbf{0 . 0 9 7}(0.025)$ & $0.231(0.144)$ & $\mathbf{0 . 3 0 2}(0.072)$ & $\mathbf{6 1 6 , 7 1 9}(13,581)$ & $\mathbf{- 3 . 8 5 9}(0.085)$ \\
\hline ALP-SB & $0.125(0.019)$ & $0.439(0.115)$ & $0.398(0.075)$ & $617,165(13,543)$ & $-3.864(0.085)$ \\
\hline SLP-SB & $0.144(0.031)$ & $0.421(0.113)$ & $0.520(0.143)$ & $617,280(13,526)$ & $-3.865(0.085)$ \\
\hline BayesReg & $0.187(0.032)$ & - & $0.695(0.169)$ & $617,529(13,513)$ & $-3.868(0.085)$ \\
\hline edgeR-L & $0.222(0.021)$ & - & - & - & - \\
\hline edgeR-Q & $0.205(0.020)$ & - & - & - & - \\
\hline BhGLM & $0.266(0.039)$ & - & - & - & - \\
\hline
\end{tabular}

(b) Parameter Estimation and Model Fit

Table 7: [Simulation 6: Comparison] Results from the simulation study with $N=200$ samples taken at $n=100$ time points with $J=400$ OTUs and $P=10$ covariates. 


\begin{tabular}{rccccc}
\hline Model & MCC & ACC & AUC & Brier-Score & $\mathrm{F}_{1}$ \\
\hline ANLP-SB & $\mathbf{0 . 7 5 2}(0.112)$ & $\mathbf{0 . 8 7 8}(0.056)$ & $\mathbf{0 . 9 3 7}(0.051)$ & $\mathbf{0 . 2 1 3}(0.108)$ & $\mathbf{0 . 8 7 8}(0.056)$ \\
ALP-SB & $0.334(0.075)$ & $0.609(0.099)$ & $0.873(0.017)$ & $0.618(0.156)$ & $0.705(0.095)$ \\
SLP-SB & $0.314(0.079)$ & $0.591(0.107)$ & $0.894(0.018)$ & - & $0.698(0.098)$ \\
BayesReg & $0.681(0.058)$ & $0.837(0.037)$ & $0.877(0.018)$ & - & $0.815(0.020)$ \\
edgeR-L & $-0.026(0.104)$ & $0.502(0.060)$ & $0.484(0.061)$ & - & $0.378(0.105)$ \\
edgeR-Q & $-0.032(0.095)$ & $0.498(0.058)$ & $0.480(0.058)$ & - & $0.399(0.092)$ \\
BhGLM & $0.437(0.051)$ & $0.678(0.069)$ & $0.766(0.028)$ & - & $0.533(0.031)$ \\
\hline
\end{tabular}

(a) Variable Selection

\begin{tabular}{|c|c|c|c|c|c|}
\hline \multirow{2}{*}{ Model } & \multicolumn{3}{|c|}{ RMSE } & \multirow{2}{*}{ DIC } & \multirow{2}{*}{ LPML } \\
\hline & $\beta_{j p}$ & $\pi_{p 0}^{\star}$ & $g_{t k j}$ & & \\
\hline ANLP-SB & $0.111(0.024)$ & $\mathbf{0 . 2 2 4}(0.099)$ & $0.426(0.102)$ & 633,665 $(18,953)$ & $\mathbf{- 3 . 9 7 1}(0.119)$ \\
\hline ALP-SB & $0.126(0.011)$ & $0.470(0.083)$ & $0.495(0.084)$ & $634,361(18,864)$ & $-3.979(0.118)$ \\
\hline SLP-SB & $0.145(0.025)$ & $0.452(0.081)$ & $0.800(0.268)$ & $634,656(18,839)$ & $-3.981(0.118)$ \\
\hline BayesReg & $0.183(0.026)$ & - & $1.088(0.302)$ & $635,145(18,811)$ & $-3.989(0.118)$ \\
\hline edgeR-L & $0.245(0.017)$ & - & - & - & - \\
\hline edgeR-Q & $0.224(0.017)$ & - & - & - & - \\
\hline BhGLM & $0.291(0.032)$ & - & - & - & - \\
\hline
\end{tabular}

(b) Parameter Estimation and Model Fit

Table 8: [Simulation 7: Comparison] Results from simulation study with $N=200$ samples taken at $n=100$ time points with $J=400$ OTUs and $P=20$ covariates.

\begin{tabular}{rccccc}
\hline Model & MCC & ACC & AUC & Brier-Score & $\mathrm{F}_{1}$ \\
\hline ANLP-SB & $\mathbf{0 . 7 4 4}(0.033)$ & $\mathbf{0 . 8 6 6}(0.023)$ & $\mathbf{0 . 9 5 9}(0.007)$ & $\mathbf{0 . 2 3 6}(0.041)$ & $\mathbf{0 . 8 3 5}(0.015)$ \\
ALP-SB & $0.081(0.035)$ & $0.480(0.051)$ & $0.817(0.014)$ & $0.699(0.072)$ & $0.630(0.050)$ \\
SLP-SB & $0.038(0.029)$ & $0.466(0.054)$ & $0.840(0.019)$ & - & $0.627(0.051)$ \\
BayesReg & $0.645(0.032)$ & $0.813(0.026)$ & $0.850(0.013)$ & - & $0.756(0.017)$ \\
edgeR-L & $-0.001(0.048)$ & $0.522(0.035)$ & $0.498(0.029)$ & - & $0.334(0.051)$ \\
edgeR-Q & -0.015() 0.050 & $0.515(0.038)$ & $0.491(0.030)$ & - & $0.336(0.046)$ \\
BhGLM & $0.321(0.037)$ & $0.646(0.042)$ & $0.687(0.022)$ & - & $0.426(0.042)$ \\
\hline
\end{tabular}

(a) Variable Selection

\begin{tabular}{|c|c|c|c|c|c|}
\hline \multirow{2}{*}{ Model } & \multicolumn{3}{|c|}{ RMSE } & \multirow{2}{*}{ DIC } & \multirow{2}{*}{ LPML } \\
\hline & $\beta_{j p}$ & $\pi_{p 0}^{\star}$ & $g_{t k j}$ & & \\
\hline ANLP-SB & $0.171(0.022)$ & $0.249(0.023)$ & $1.087(0.261)$ & $334,422(15,563)$ & $-4.211(0.196)$ \\
\hline ALP-SB & $0.152(0.011)$ & $0.456(0.023)$ & $\mathbf{0 . 6 5 5}(0.078)$ & $334,196(15,512)$ & $-4.232(0.195)$ \\
\hline SLP-SB & $0.153(0.017)$ & $0.449(0.023)$ & $1.120(0.323)$ & $334,599(15,495)$ & $-4.238(0.195)$ \\
\hline BayesReg & $0.185(0.016)$ & - & $1.742(0.353)$ & $\mathbf{3 3 4 , 1 6 1}(15,526)$ & $-4.252(0.195)$ \\
\hline edgeR-L & $0.344(0.022)$ & - & - & - & - \\
\hline edgeR-Q & $0.320(0.021)$ & - & - & - & - \\
\hline BhGLM & $0.423(0.035)$ & - & - & - & - \\
\hline
\end{tabular}

(b) Parameter Estimation and Model Fit

Table 9: [Simulation 8: Comparison] Results from simulation study with $N=200$ samples taken at $n=100$ time points with $J=200$ OTUs and $P=50$ covariates. 
We let $\pi_{p 0}^{\star, \mathrm{TR}}=0.95$ or 0.05 with equal probability, and $\pi_{p 1}^{\mathrm{TR}} \stackrel{i i d}{\sim} \mathrm{Be}(5,10)$. We let $\gamma_{j p}^{\mathrm{TR}}=0$, 1 or 2 with probabilities, $\boldsymbol{\pi}_{p}^{\star, \mathrm{TR}}=\left(\pi_{p 0}^{\star, \mathrm{TR}},\left(1-\pi_{p 0}^{\star, \mathrm{TR}}\right) \pi_{p 1}^{\mathrm{TR}},\left(1-\pi_{p 0}^{\star, \mathrm{TR}}\right)\left(1-\pi_{p 1}^{\mathrm{TR}}\right)\right)$. We generated $\sigma_{p}^{2^{\mathrm{TR}}} \stackrel{i i d}{\sim} \operatorname{Unif}(3 / 10,4 / 10)$ and $\iota_{p}^{\mathrm{TR}} \stackrel{i i d}{\sim} \operatorname{Unif}(1 / 10,3 / 10)$. We simulated $\beta_{j p}^{\mathrm{TR}}$ conditional on $\gamma_{j p}^{\mathrm{TR}}$; if $\gamma_{j p}^{\mathrm{TR}}=0$, then $\beta_{j p}^{\mathrm{TR}}=0$. For the cases of $\gamma_{j p}^{\mathrm{TR}} \neq 0$, we generated $\beta_{j p}^{\mathrm{TR}}$ from the normal distributions with mean 0 and variance $\sigma_{p}^{2 \text {, TR }}$ truncated from below at $\iota_{p}^{\mathrm{TR}} \sigma_{p}^{\mathrm{TR}}$ if $\gamma_{j p}^{\mathrm{TR}}=1$ and from above at $-\iota_{p}^{\mathrm{TR}} \sigma_{p}^{\mathrm{TR}}$ if $\gamma_{j p}^{\mathrm{TR}}=2$. We kept the same simulation setup for $\alpha_{t j}^{\mathrm{TR}}$ and $s_{j}$. We then drew the OTU counts from $Y_{t k j} \mid \mu_{t k j}^{\mathrm{TR}}, s_{j}^{\mathrm{TR}} \stackrel{i n d e p}{\sim} \operatorname{NB}\left(\mu_{t k j}^{\mathrm{TR}}\left(\boldsymbol{x}_{t}\right), s_{j}^{\mathrm{TR}}\right)$, where $\mu_{t k j}^{\mathrm{TR}}\left(\boldsymbol{x}_{t}\right)=\exp \left(r_{t k}^{\mathrm{TR}}+\alpha_{0 j}^{\mathrm{TR}}+\alpha_{t j}^{\mathrm{TR}}+\boldsymbol{x}_{t}^{\prime} \boldsymbol{\beta}_{j}^{\mathrm{TR}}\right)$. A total of 100 datasets were simulated under each scenario. The models, including ANLP-SB and the competing models, are compared under eight criteria. Tables 5-9 summarize results for Scenarios 4-8, respectively. The averages for the metrics are listed in the table along with standard deviations in parenthesis. Under all scenarios, ANLP-SB outperforms the other models in terms of variable selection. For Scenarios 4-7, ANLP-SB yields better parameter estimates as well. In Simulation 8, ALP-SB and SLP-SB obtain better RMSE for $\beta_{j p}$, ALP-SB for RMSE for $g_{t k j}$, and BayeReg for DIC, while ANLP-SB is still very close to the best performers under those criteria. The results demonstrate that ANLP-SB is well-suited for scaling up to higher dimensional settings.

The last column of Table 4 lists the run-times in minutes for 1,000 MCMC iterations using the setups from Simulations 1-8. Run times of ANLP-SB depend on the size of the data $(J, P, n, N)$ as well as some fixed hyperparameters $\left(L^{r}, L^{\alpha}, M\right)$. The number of time points $(n)$ and replicates at a time point $\left(K_{i}\right)$ determine the total number of samples $(N)$. Comparing the run times of Simulation 4 and 5 to those of Simulations 6 and 7 , respectively, shows the impact of an increase in $n$ on the computational cost. The number of sample-specific size factors $r_{t k}$ increases in $N$, and updating them involves updating two mixture indicators, $c_{t k}^{r}$ and $\lambda_{t k}^{r}$ for each $r_{t k}$, potentially resulting in a substantial increase in the computational cost. The computational cost for $\alpha_{0 j}$ scales in a similar way to that of $r_{t k}$, but with respect to the number of OTUs $J$. Another factor that may significantly increase the computational cost of the model is the number of candidate covariates, $P$ especially with large $J$, as indicated from comparing run times of Simulations 4 and 6 to those of Simulations 5 and 7 , respectively. The computation time for $\beta_{j p}$ and $\gamma_{j p}$ scales with both $J$ and $P$. A large $P$ also increases the number of other parameters such as $\sigma_{p}^{2}, \iota_{p}$ and $\boldsymbol{\pi}_{p}$. The amount of computation required may rapidly escalate with increasing $P$ when $J$ is large. We note that compared to $N$ and $J$, the values of $L^{r}, L^{\alpha}$, and $M$ do not significantly impact run times because they are related to hyperparameters at a high level of the model. Also, parameters $\alpha_{t j}=\sum_{m=1}^{M} K\left(t-u_{m}\right) \theta_{m j}$ are deterministically calculated given $\theta_{m j}$ and prespecified kernel $K$, and the computation time for $\alpha_{t j}$ scales with $M$ and $J$.

\section{Additional Results for Ocean Microbiome Data Analysis}

In this section, we present additional results from the ocean microbiome data analysis in $\S 4$ of the main text. Table 10 shows the names of the covariates. Figure 7 has 


\begin{tabular}{lll}
\hline Covariate Name & Short Name & Levels \\
\hline Alexandrium $\left(x_{1}-x_{3}\right)$ & $\mathrm{Ax}$ & low/medium/high \\
Dinophysis $\left(x_{4}-x_{6}\right)$ & $\mathrm{Dp}$ & low/medium/high \\
Pseudo-nitzschia $\left(x_{7}-x_{10}\right)$ & $\mathrm{Pn}$ & low/medium/high/very high \\
Domoic acid $\left(x_{11}-x_{14}\right)$ & $\mathrm{DA}$ & low/medium/high/very high \\
Ammonia $\left(x_{15}\right)$ & $\mathrm{NH}_{4}$ & continuous \\
Nitrate $\left(x_{16}\right)$ & $\mathrm{N}$ & continuous \\
Phosphate $\left(x_{17}\right)$ & $\mathrm{P}$ & continuous \\
Silicate $\left(x_{18}\right)$ & $\mathrm{Si}$ & continuous \\
Water Temperature $\left(x_{19}\right)$ & $\mathrm{T}$ & continuous \\
Chlorophyll $\left(x_{20}\right)$ & $\mathrm{Chl}$ & continuous \\
\hline
\end{tabular}

Table 10: Covariate names in the ocean microbiome dataset. Categories are listed for the discretized covariates.

\begin{tabular}{rcc}
\hline Model & DIC & LPML \\
\hline ANLP-SB & $\mathbf{2 5 6 , 1 8 9}$ & $\mathbf{- 3 . 2 5 2}$ \\
ALP-SB & 256,238 & -3.254 \\
SLP-SB & 256,330 & -3.254 \\
BayesReg & 267,010 & -3.609 \\
\hline
\end{tabular}

Table 11: Performance metrics of the Bayesian models applied to the ocean microbiome dataset. Best performances are in bold.

simplex plots of the posterior probability vectors $\hat{z}_{j p}=\left(\hat{z}_{j p 0}, \hat{z}_{j p 1}, \hat{z}_{j p 2}\right)$, where $\hat{z}_{j p q}$ is an posterior estimate of the probability that $\gamma_{j p}=q, q \in\{0,1,2\}$ for the complete set of covariates under ANLP-SB. Each point on the simplex plot represents the posterior probabilities of no effect (bottom left tip)/positive effect (apex)/negative effect (bottom right tip) of that covariate for an OTU. These plots provide insights into how the covariates are associated with community dynamics. For some covariates the effect directions on the OTU abundances are relatively homogeneous, while other covariates have more varied effects. Figure 8 illustrates posterior inference for the OTUs belonging to class Gamma-proteobacteria. The figure has histograms of posterior estimates of regression coefficients $\left(\hat{\beta}_{j p}\right)$ and probabilities of $\gamma_{j p}=2\left(\hat{z}_{j p 2}\right)$ for the different DA concentration levels. Figure 9 shows results on bacterial growth of a lab experiment using a cultured Gamma-proteobacteria strain. The results show that the bacteria was significantly affected by DA at concentrations ranging from 25 to $50 \mu \mathrm{g} / \mathrm{ml}$, which validates our findings from Figure 8. To assess the convergence of the posterior MCMC simulation, we re-ran the data analysis under the six different hyperparameter specifications given in Table 1. The trace plots in Figure 10 illustrate that the log-likehood converges to similar states under the different prior specifications and different random seeds. The results provide practical evidence of posterior convergence and indicate robustness of ANLP-SB to the prior specification.

For comparison, we applied the competing models to the ocean microbiome data. Figure 11(a)-(b) have the posterior distributions of $\pi_{p 0}^{\star}$ probabilities that a covariate has no effect on OTU abundance and of $\pi_{p 1}$ conditional probabilities that a covariate 
has a positive effect given it has a significant effect under ALP-SB, respectively. Figure 11(c) shows posterior distributions of $\pi_{p 0}^{\star}$ under SLP-SB. The posterior distributions of $\pi_{p 0}^{\star}$ and $\pi_{p 1}$ under ANLP-SB are shown in Figure 4 of the main text. The models with local priors have posterior mean estimates of $\pi_{p 0}^{\star}$ between 0.2 and 0.4 for all covariates, while those values are above or around 0.5 under ANLP-SB. The ANLP induces more sparsity than the ALP and the SLP, resulting in more parsimonious models. Table 11 has DIC and LPML for the Bayesian models. ANLP-SB has the best fit according to both criteria, followed by ALP-SB, and then SLP-SB. Figure 12 shows the proportion of OTUs that have a significant relationship with each covariate, where the criteria used to define a variable as 'selected' is the same as was described in $\S 3$ of the main text. For ANLP-SB, a variable is 'selected' when $\mathrm{P}\left(\gamma_{j p} \neq 0 \mid \boldsymbol{Y}\right)>0.5$. For BayesReg, the $95 \%$ posterior credible intervals were used to select a variable. For the frequentist models, we selected a variable when the adjusted p-value for that regression coefficient was less than 0.05 .

\section{References}

Carlin, B. P. and Chib, S. (1995). "Bayesian Model Choice via Markov Chain Monte Carlo Methods." Journal of the Royal Statistical Society, 57(3): 473-484.

URL www.jstor.org/stable/2346151 2

Li, Q., Guindani, M., Reich, B. J., Bondell, H. D., and Vannucci, M. (2017). "A Bayesian mixture model for clustering and selection of feature occurrence rates under mean constraints." Statistical Analysis and Data Mining: The ASA Data Science Journal, 10(6): 393-409.

URL http://dx.doi.org/10.1002/sam.113502 


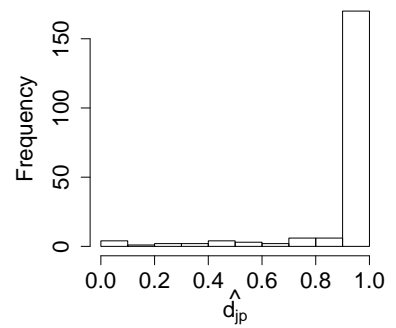

(a) Case 1: $(1,1,2.5,10)$

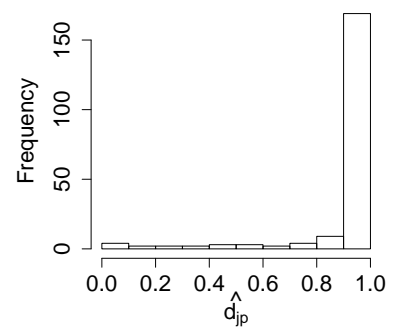

(d) Case 4: $(0.1,0.1,1,10)$

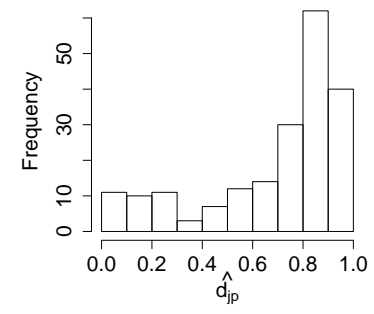

(g) Case 1: $(1,1,2.5,10)$

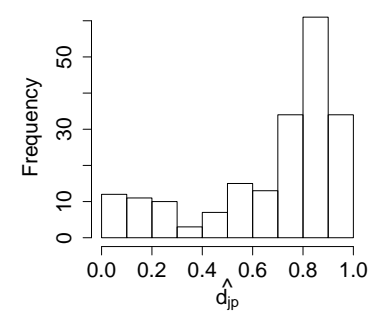

(j) Case 4: $(0.1,0.1,1,10)$

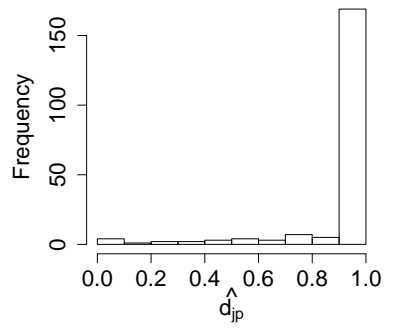

(b) Case 2: $(0.1,0.1,2.5,10)$

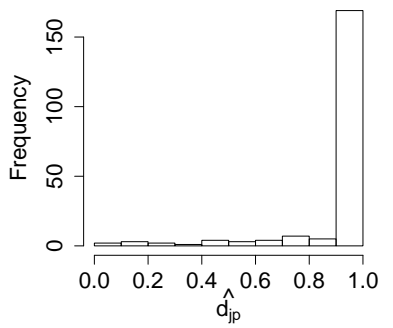

(e) Case 5: $(1,1,5,20)$

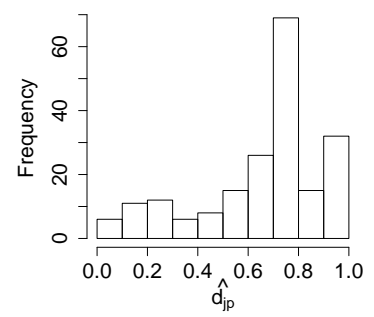

(h) Case 2: $(0.1,0.1,2.5,10)$

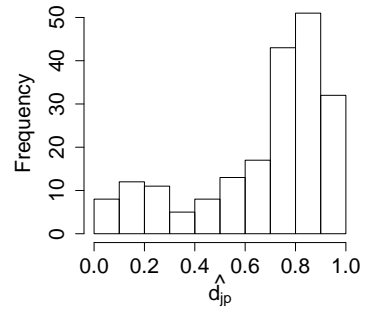

(k) Case 5: $(1,1,5,20)$

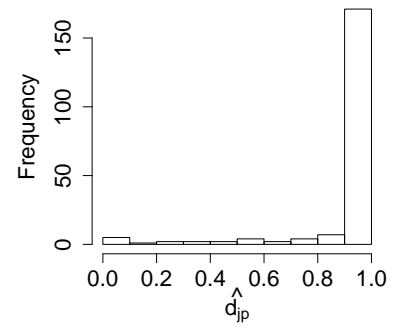

(c) Case 3: (1, 1, 1, 10)

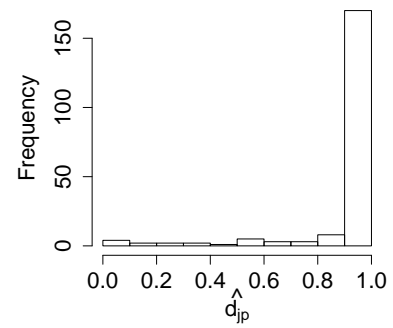

(f) Case 6: $(0.1,0.1,5,10)$

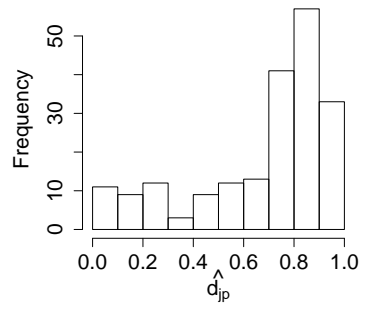

(i) Case 3: $(1,1,1,10)$

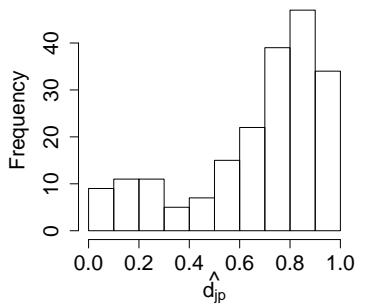

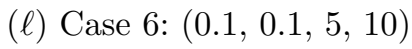

Figure 4: [Simulation 1] Histograms of posterior estimates of $\hat{d}_{j p}=\hat{\mathrm{P}}\left(\gamma_{j p}=\gamma_{j p}^{\mathrm{TR}}\right)$ for selected covariates $x_{1}$ and $x_{5}$ under the six different specifications of $\left(a_{\iota}, b_{\iota}, a_{\sigma}, b_{\sigma}\right)$ in Table 1. Panels (a)-(f) show results from $x_{1}$ (Silicate), and panels $(\mathrm{g})-(\ell)$ show results from $x_{5}$ (low concentration of Alexandrium). 


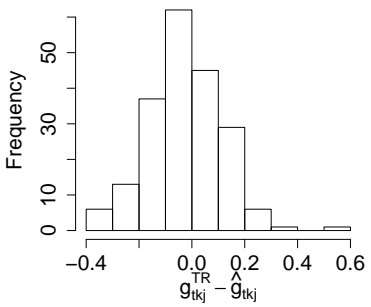

(a) $v \mp 2, m=70$

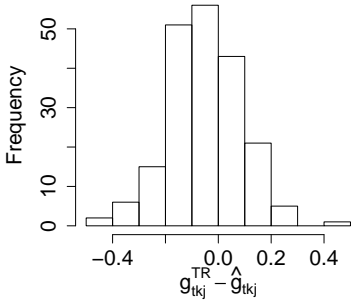

(c) $m=90$

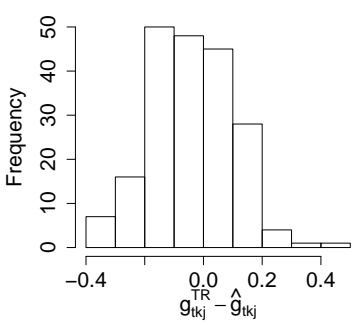

(b) $v \pm 2, m=70$

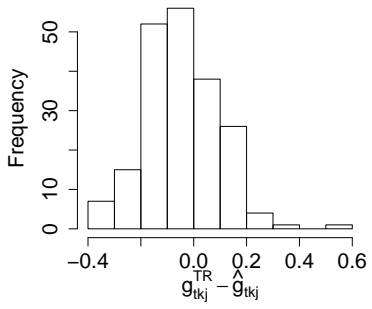

(d) $m=50$

Figure 5: [Simulation 1] Histograms of differences between the true baseline mean counts and their estimates, $g_{t k j}^{\mathrm{TR}}-\hat{g}_{t k j}$, under different specifications for $v_{r}, v_{\alpha}$ and $M$. 

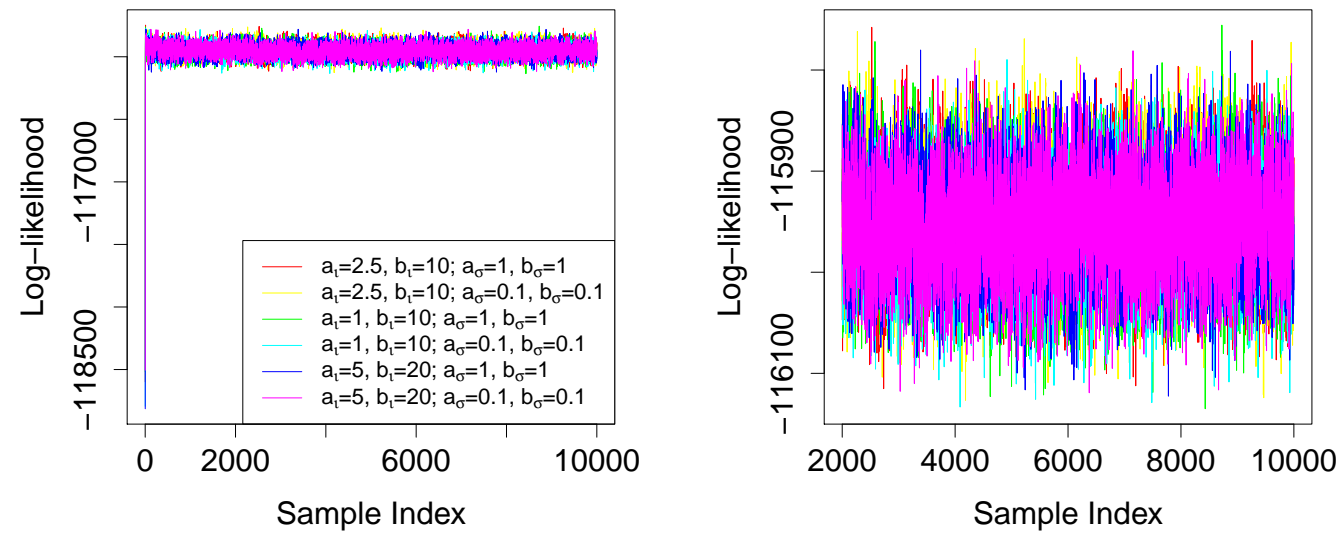

Figure 6: [Simulation 1] Trace plots of the log-likelihood under different prior specifications. The plots are over the course of the entire MCMC (left), and after 2,000 samples (right). 


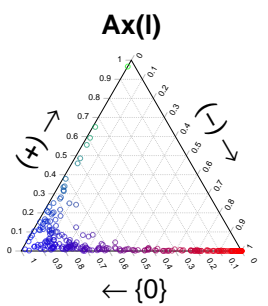

(a) Alexandrium(l)

$\mathrm{Dp}(\mathrm{m})$

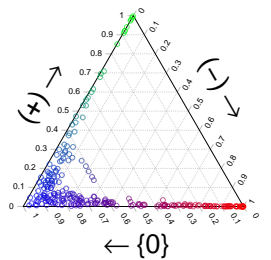

(e) Dinophysis(m)

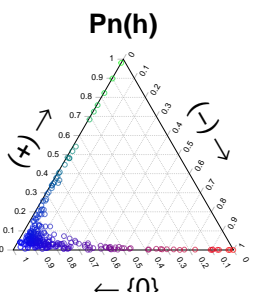

(i) Pseudo-nitzschia(h)

DA(h)

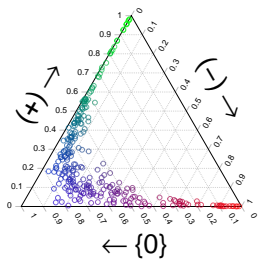

(m) Domoic acid(h)

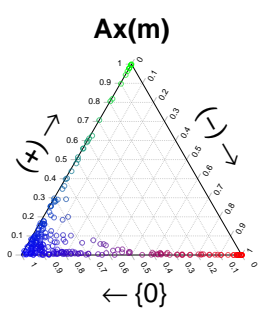

(b) Alexandrium (m)

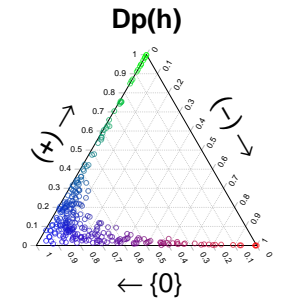

(f) Dinophysis(h)

$\mathrm{Pn}(\mathrm{H})$

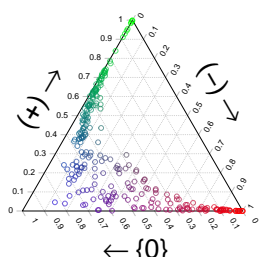

(j) Pseudo-nitzschia $(\mathrm{H})$

DA(H)

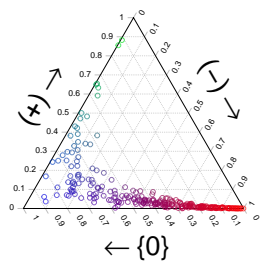

(n) Domoic $\operatorname{acid}(\mathrm{H})$

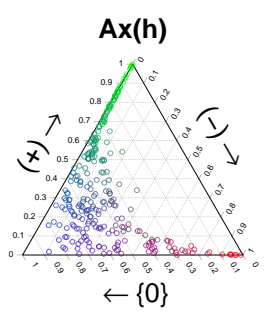

(c) Alexandrium(h)

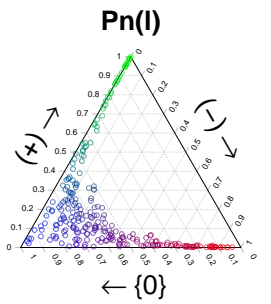

(g) Pseudo-nitzschia(l)

DA(I)

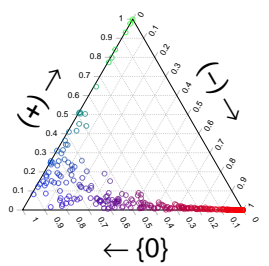

(k) Domoic acid(l)

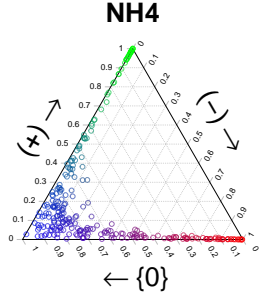

(o) Ammonia

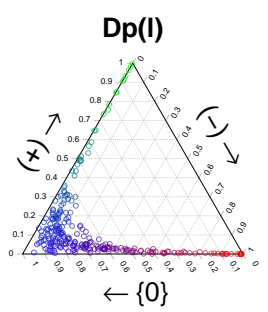

(d) Dinophysis(l) Pn(m)

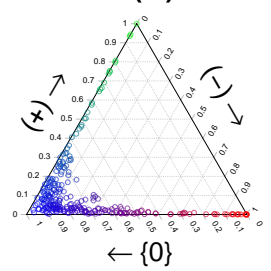

(h) Pseudo-nitzschia(m)

DA(m)

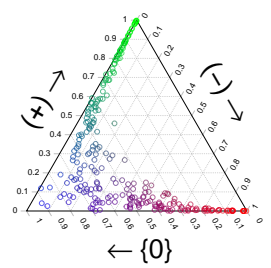

(l) Domoic $\operatorname{acid}(\mathrm{m})$

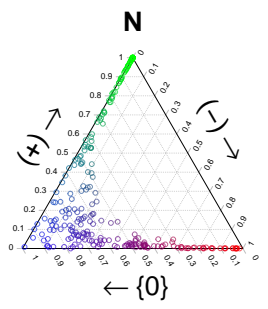

(p) Nitrate

Figure 7: [Ocean Microbiome Data] Simplex plots of the posterior means $\hat{z}_{j p}=\left(\hat{z}_{j p 0}, \hat{z}_{j p 1}, \hat{z}_{j p 2}\right)$ of $\gamma_{j p}=0$, (no effect), $\gamma_{j p}=1$, (positive effect) and $\gamma_{j p}=2$, (negative effect). The colors, blue, red and green, indicate no relationship, a negative relationship, and a positive relationship with OTU abundances, respectively. 


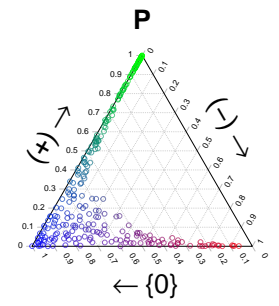

(q) Phosphate

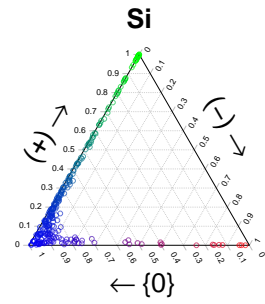

(r) Silicate

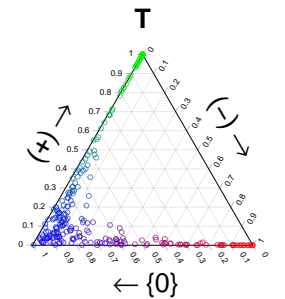

(s) Water Temperature

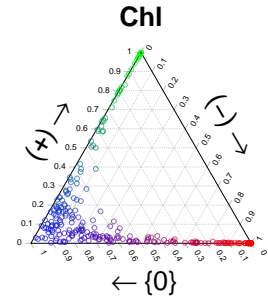

(t) Chlorophyll

[Ocean Microbiome Data - Supplementary Figure 7 continued] Simplex plots of the posterior means $\hat{\boldsymbol{z}}_{j p}=\left(\hat{z}_{j p 0}, \hat{z}_{j p 1}, \hat{z}_{j p 2}\right)$ of $\gamma_{j p}=0$, (no effect), $\gamma_{j p}=1$, (positive effect) and $\gamma_{j p}=2$, (negative effect). The colors, blue, red and green, indicate no relationship, a negative relationship, and a positive relationship with OTU abundances, respectively.

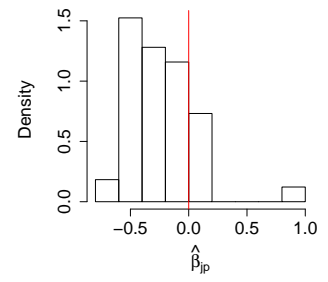

(a) $\hat{\beta}$ for $\mathrm{DA}(\mathrm{l})$

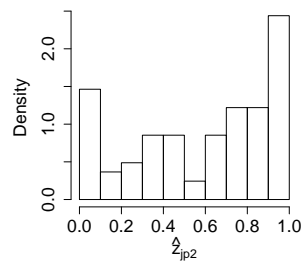

(e) $\hat{z}_{2}$ for $\mathrm{DA}(\mathrm{l})$

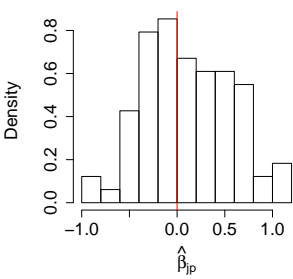

(b) $\hat{\beta}$ for $\mathrm{DA}(\mathrm{m})$

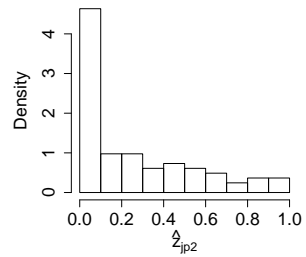

(f) $\hat{z}_{2}$ for $\mathrm{DA}(\mathrm{m})$

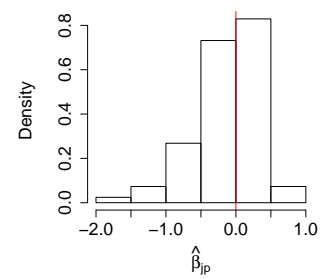

(c) $\hat{\beta}$ for $\mathrm{DA}(\mathrm{h})$

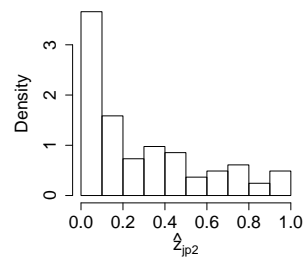

(g) $\hat{z}_{2}$ for $\mathrm{DA}(\mathrm{h})$

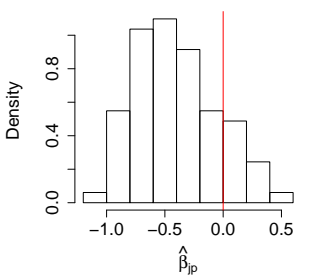

(d) $\hat{\beta}$ for $\mathrm{DA}(\mathrm{H})$

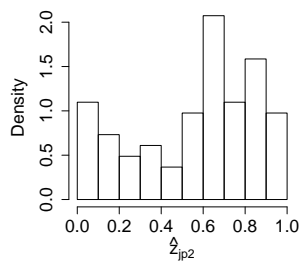

(h) $\hat{z}_{2}$ for $\mathrm{DA}(\mathrm{H})$

Figure 8: [Ocean Microbiome Data] Histograms of $\hat{\beta}_{j p}$ and $\hat{z}_{j p 2}$ for different DA concentration levels for OTUs belonging to the class Gamma-proteobacteria. 


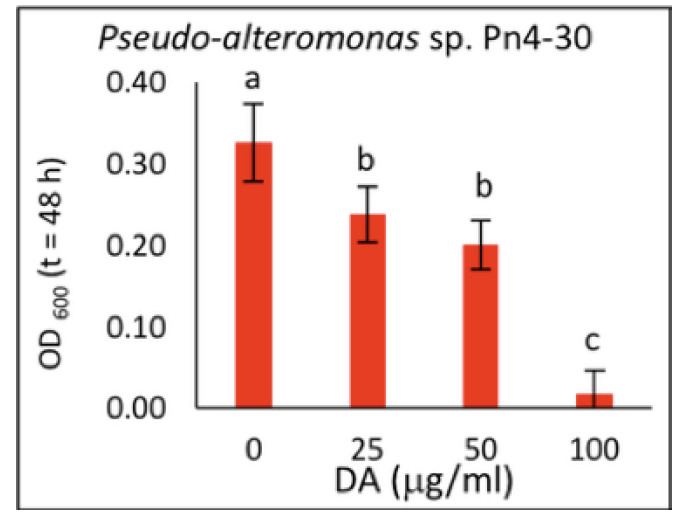

Figure 9: [Ocean Microbiome Data] Plots of growth measurements (Optical Density at $600 \mathrm{~nm}$ ) of a bacterial cultured isolate belonging to Gamma-proteobacteria measured after 48 hours of exposure to different concentration levels of domoic acid (DA).
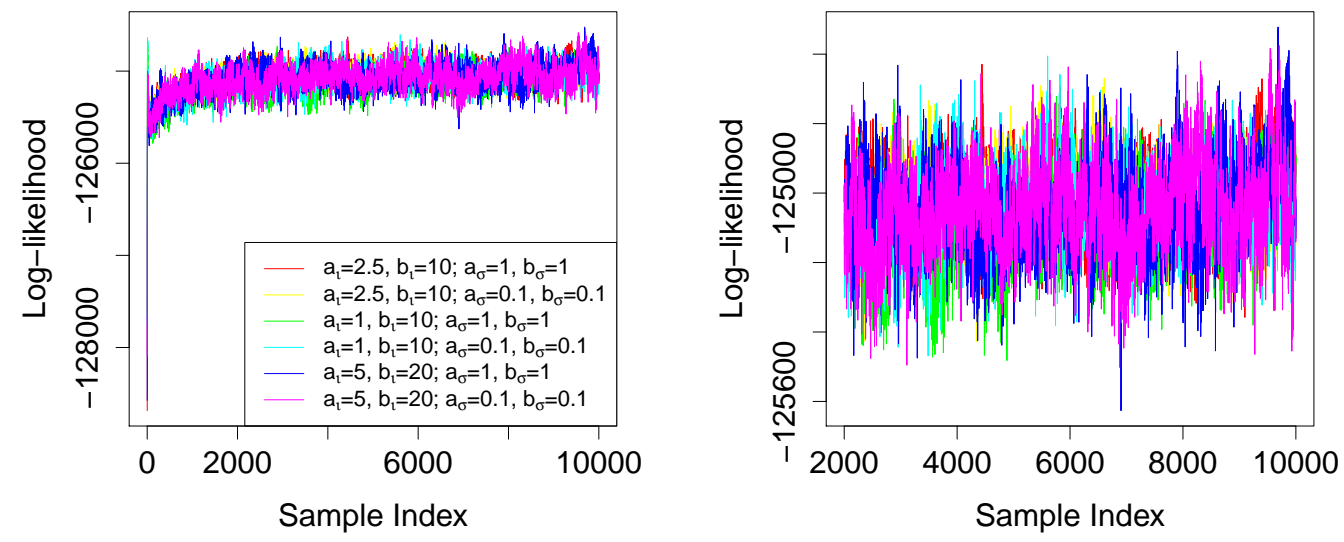

Figure 10: [Ocean Microbiome Data] Trace plots of the log-likelihood under different prior specifications. The plots are over the course of the entire MCMC (left), and after 2,000 samples (right). 


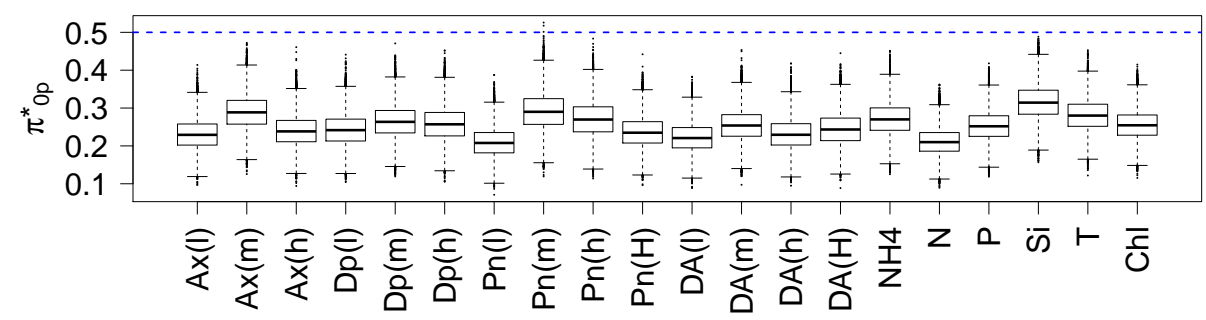

(a) Posterior distributions of $\pi_{p 0}^{\star}$ under ALP-SB

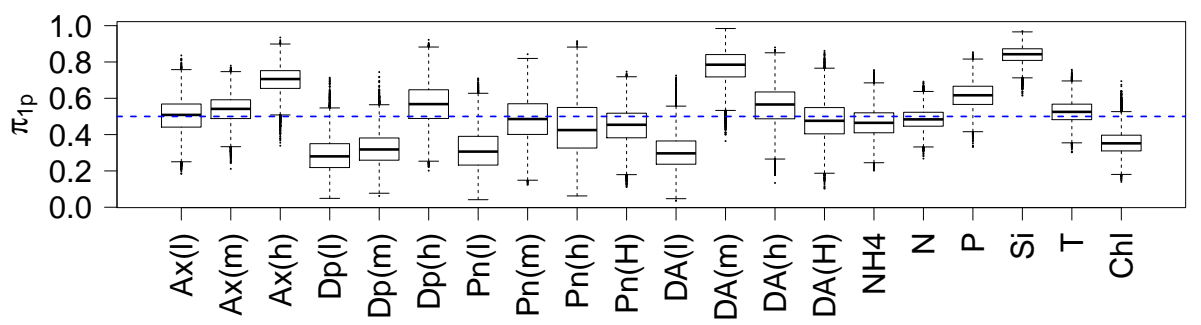

(b) Posterior distributions of $\pi_{p 1}$ under ALP-SB

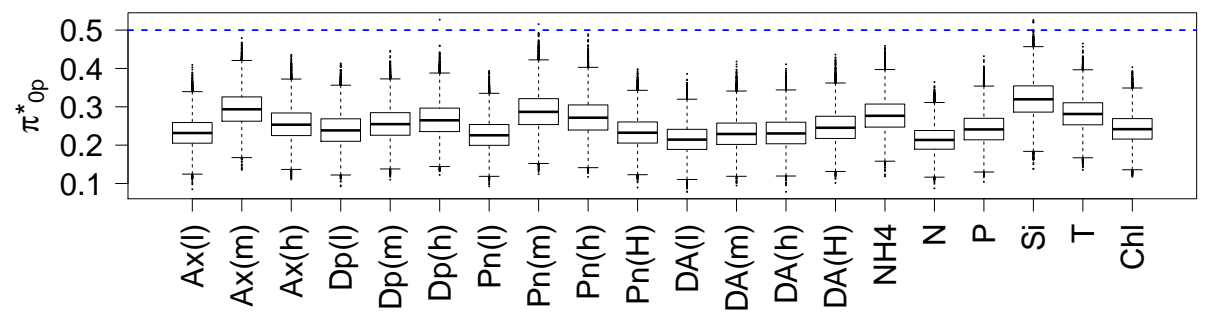

(c) Posterior distributions of $\pi_{p 0}^{\star}$ under SLP-SB

Figure 11: [Ocean Microbiome Data] Panels (a) and (c): Boxplots of the posterior distributions of $\pi_{p 0}^{\star}$, the probability of a non-zero effect on OTU abundance, under ALP-SB and SLP-SB, respectively. Panel (b): Boxplots of the posterior distributions of $\pi_{p 1}$, the conditional probability of a positive effect direction given the covariate has a non-zero effect under ALP-SB. 

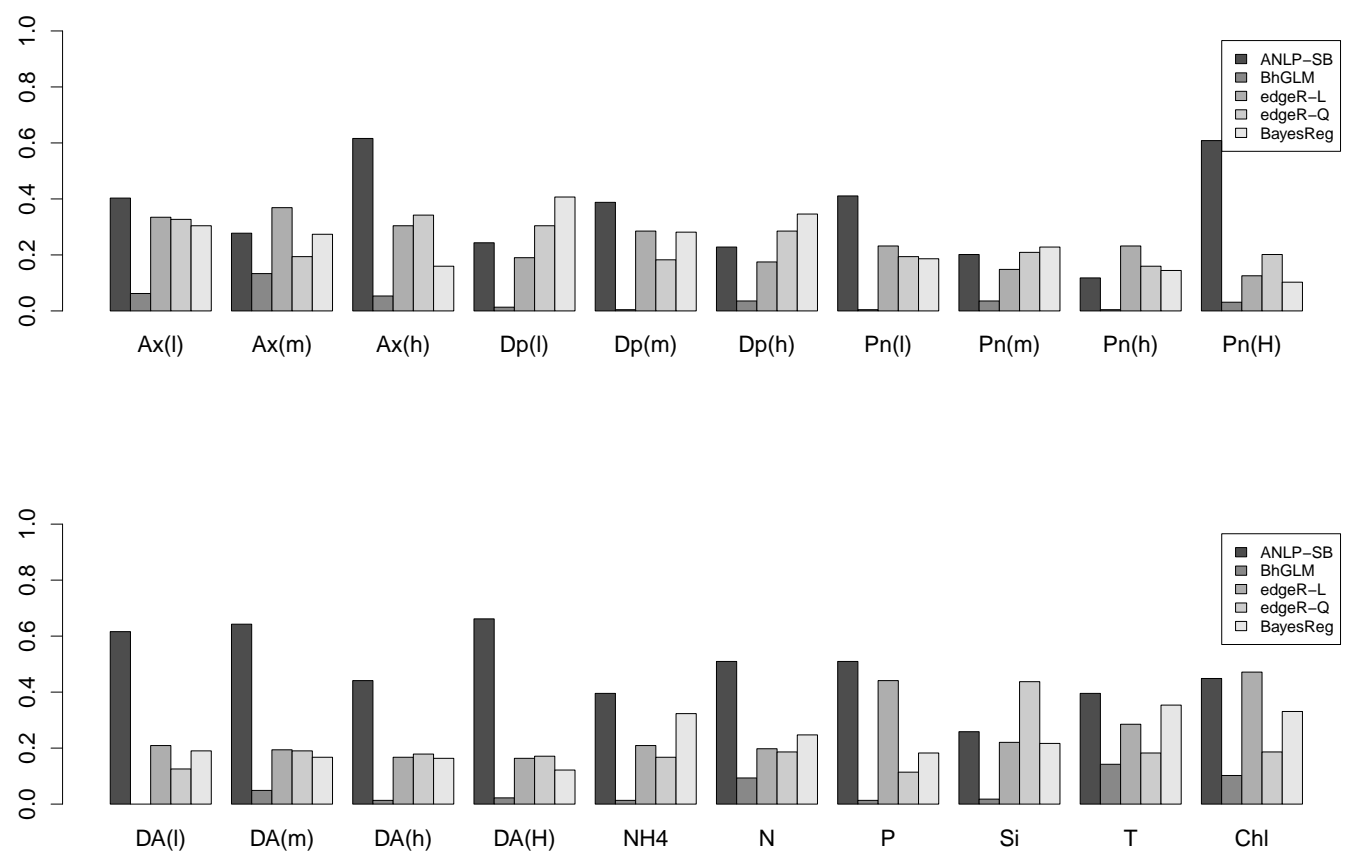

Figure 12: [Ocean Microbiome Data] Proportions that each covariate is selected under ANLP-SB, BayesReg, BhGLM, edgeR-L and edgeR-Q. 